\title{
SPATIALLY RESOLVED STELLAR, DUST, AND GAS PROPERTIES OF THE POST-INTERACTING WHIRLPOOL GALAXY SYSTEM
}

\author{
Erin Mentuch Cooper ${ }^{1,2}$, Christine D. Wilson ${ }^{1}$, Kelly $_{\text {Foyle }}{ }^{1}$, George Bendo ${ }^{3}$, Jin Koda ${ }^{4}$, Maarten Baes ${ }^{5}$, \\ Médéric Boquien $^{6}$, Alessandro Boselli $^{6}$, Laure Ciesla ${ }^{6}$, Asantha Cooray ${ }^{7}$, Steve Eales $^{8}$, Maud Galametz $^{9}$, \\ Vianney Lebouteiller ${ }^{10}$, Tara Parkin $^{1}$, Hélène Roussel ${ }^{11}$, MarC SAuvage $^{10}$, \\ Luigi SPINOGLIO $^{12}$, AND MatThew W. L. SMith ${ }^{8}$ \\ ${ }^{1}$ Department of Physics \& Astronomy, McMaster University, Hamilton, ON L8S 4M1, Canada \\ ${ }^{2}$ Astronomy Department, University of Texas at Austin, Austin, TX 78712, USA \\ ${ }^{3}$ UK ALMA Regional Center Node, Jodrell Bank Center for Astrophysics, School of Physics \& Astronomy, University of Manchester, \\ Oxford Road, Manchester M13 9PL, UK \\ ${ }^{4}$ Department of Physics \& Astronomy, SUNY Stony Brook, Stony Brook, NY 11794-3800, USA \\ ${ }^{5}$ Sterrenkundig Observatorium, Universiteit Gent, Krijgslaan 281 S9, B-9000 Gent, Belgium \\ ${ }^{6}$ Laboratoire d'Astrophysique de Marseille, UMR 6110 CNRS, 38 rue F. Joliot-Curie, F-13388 Marseille, France \\ ${ }^{7}$ Department of Physics \& Astronomy, University of California, Irvine, 4186 Frederick Reines Hall, Irvine, CA 92697, USA \\ ${ }^{8}$ School of Physics \& Astronomy, Cardiff University, The Parade, Cardiff CF24 3AA, UK \\ ${ }^{9}$ Institute of Astronomy, University of Cambridge, Madingley Road, Cambridge CB3 OHA, UK \\ ${ }^{10}$ CEA/DSM/DAPNIA/Service d'Astrophysique, UMR AIM, CE Saclay, F-91191 Gif sur Yvette Cedex, France \\ ${ }^{11}$ Institut d'Astrophysique de Paris, UMR7095 CNRS, Université Pierre \& Marie Curie, 98 bis Boulevard Arago, F-75014 Paris, France \\ ${ }^{12}$ Istituto di Fisica dello Spazio Interplanetario INAF, Via Fosso del Cavaliere 100, I-00133 Roma, Italy \\ Received 2012 March 2; accepted 2012 June 13; published 2012 August 7
}

\begin{abstract}
Using infrared imaging from the Herschel Space Observatory, observed as part of the Very Nearby Galaxies Survey, we investigate the spatially resolved dust properties of the interacting Whirlpool galaxy system (NGC 5194 and NGC 5195), on physical scales of $\sim 1 \mathrm{kpc}$. Spectral energy distribution modeling of the new infrared images in combination with archival optical and near- through mid-infrared images confirms that both galaxies underwent a burst of star formation 370-480 Myr ago and provides spatially resolved maps of the stellar and dust mass surface densities. The resulting average dust-to-stellar mass ratios are comparable to other spiral and spheroidal galaxies studied with Herschel, with NGC 5194 at $\log \left(M_{\text {dust }} / M_{\star}\right)=-2.5 \pm 0.2$ and NGC 5195 at $\log \left(M_{\text {dust }} / M_{\star}\right)=-3.5 \pm 0.3$. The dust-to-stellar mass ratio is constant across NGC 5194 suggesting the stellar and dust components are coupled. In contrast, the mass ratio increases with radius in NGC 5195 with decreasing stellar mass density. Archival mass surface density maps of the neutral and molecular hydrogen gas are also folded into our analysis, revealing a fairly constant gas-to-dust mass ratio, $94 \pm 17$ across the system. Somewhat surprisingly, we find the dust in NGC 5195 is heated by a strong interstellar radiation field (ISRF), over 20 times that of the ISRF in the Milky Way, resulting in relatively high characteristic dust temperatures $(\sim 30 \mathrm{~K})$. This post-starburst galaxy contains a substantial amount of low-density molecular gas and displays a gas-to-dust ratio (73 \pm 35$)$ similar to spiral galaxies. It is unclear why the dust in NGC 5195 is heated to such high temperatures as there is no star formation in the galaxy and its active galactic nucleus is 5-10 times less luminous than the one in NGC 5194, which exhibits only a modest enhancement in the amplitude of its ISRF.
\end{abstract}

Key words: galaxies: fundamental parameters - galaxies: general - galaxies: individual (M51a/M51b) - galaxies: stellar content - infrared: galaxies - techniques: photometric

Online-only material: color figures

\section{INTRODUCTION}

The fundamental building blocks of a galaxy-its stars, gas, and dust-are expressed across its spectral energy distribution (SED). Hot gas $\left(>10^{6} \mathrm{~K}\right)$ emits brightly at very short wavelengths in the X-ray and ultraviolet (UV), while neutral and molecular gas are seen through emission lines primarily studied in the submillimeter and radio. Young stellar populations dominate the UV and blue visible light of a galaxy and ionize gas leading to numerous emission lines in the UV, optical, and infrared. Evolved stars peak at longer wavelengths in the red visible and near-infrared regime. At longer wavelengths and lower energies, mid-infrared light traces very small $(<0.01 \mu \mathrm{m})$ dust grains and small organic molecules known as polycyclic aromatic hydrocarbons (PAHs) and far-infrared light traces reprocessed stellar light emitted by dust grains. Multi-wavelength studies provide the synergy to compare these components and move toward building models that incorporate all the building blocks of a galaxy.

Standard stellar population techniques can tell us how much stellar mass is in a galaxy, its metallicity, the amount of dust attenuation, and can even shed light on the history of star formation within a galaxy. Fundamentally, stellar emission models are built from the superposition of multiple simple stellar populations (SSPs) where assumptions regarding the initial mass function (IMF) and stellar evolution lead to systematic but known uncertainties (Conroy et al. 2009). On the other hand, modeling the dust emission in a galaxy is not yet as evolved. Most dust emission models or templates (Dale \& Helou 2002; Galliano et al. 2011) measure the total dust mass and some (Désert et al. 1990; Draine \& Li 2007; Compiègne et al. 2011) measure the relative abundance of various dust emission components (e.g., PAHs, very small grains, and big grains). Fundamental to these models is the mechanism for 
dust heating, primarily provided by the interstellar radiation field (ISRF) produced by stars, although active galactic nuclei (AGNs) can also play a role in nuclear regions.

Nearby galaxies offer unique laboratories to explore how dust emission is tied to the underlying stellar populations in the context of standard SED modeling techniques. The postinteracting Whirlpool galaxy system was observed as part of the Very Nearby Galaxies Survey (VNGS; PI Christine Wilson), a guaranteed-time photometric and spectroscopic survey of a diverse range of 13 nearby galaxies with Herschel's Photodetector Array Camera and Spectrometer (PACS; Poglitsch et al. 2010) and Spectral and Photometric Imaging Receiver (SPIRE; Griffin et al. 2010) instruments.

This system consists of the diverse galaxy pair of NGC 5194/ M51a, a spiral SABbc type galaxy, and NGC 5195/M51b, a dusty spheroid galaxy whose peculiar dust lanes have given it an SB0p classification (de Vaucouleurs et al. 1995). An encounter between the two galaxies 300-500 Myr ago is inferred from both kinematic (Salo \& Laurikainen 2000) and hydrodynamic modeling (Dobbs et al. 2010) of the M51 system. In agreement, a recently constructed color-magnitude diagram of its individual stars, measured using Hubble Space Telescope (HST) archival images, suggests a burst of star formation occurred 390-450 Myr ago in NGC 5195 (Tikhonov et al. 2009). Star formation also peaked around 380-450 Myr in the spiral NGC 5194 and has continued until the present day. While star formation is ubiquitous and ongoing across NGC 5194 as shown by its $\sim 19,600 \mathrm{HII}$ regions (Lee et al. 2011) and optical colors (Tikhonov et al. 2009), the spheroid neighbor NGC 5195 shows no evidence of recent star formation through a deficiency of ionized gas (Thronson et al. 1991). This is further supported by evidence for dynamically stable molecular gas, which is unable to form stars. Kohno et al. (2002) measured both $\mathrm{CO}(1-0)$ and $\mathrm{HCN}(1-0)$ in the galaxy and found an $\mathrm{HCN}-$ to- $\mathrm{CO}$ intensity ratio 5-15 times smaller than that seen in starburst regions (Helfer \& Blitz 1993; Kohno et al. 2002) and 5 times smaller than that usually found in spiral disks (Helfer \& Blitz 1995; Kohno et al. 1999), indicative of a lack of dense molecular cores.

This paper takes advantage of a large multi-wavelength data set encompassing observations of the stars, dust, and gas at a common spatial resolution to try to decode the star formation history of each galaxy and how it has impacted the dust and gas properties. Our ultimate goal is to present a story of these components in the context of the interaction and star formation history of the system. Our observations and the steps involved to match the spatial and surface brightness distributions are given in Section 2. We apply standard SED-fitting techniques to the dust and stars separately but do so on a pixel level in order to map any gradients and spatial dependencies of the parameters. The models, our fitting procedure, and output parameter maps are all presented in Section 3. We provide dust-to-stellar and gas-to-dust mass maps and one-dimensional (1D) radial plots in Section 4 and discuss how the observed dust properties relate to the stellar and gas properties of the system.

\section{OBSERVATIONS AND IMAGE PROCESSING}

Our multi-wavelength analysis includes 20 images of the Whirlpool system, and thus exploits the great efforts of a number of large legacy surveys. In this section, we provide a summary of the archival observations and describe our new Herschel observations from the VNGS. A number of steps were taken to ensure each pixel represents a common measure of

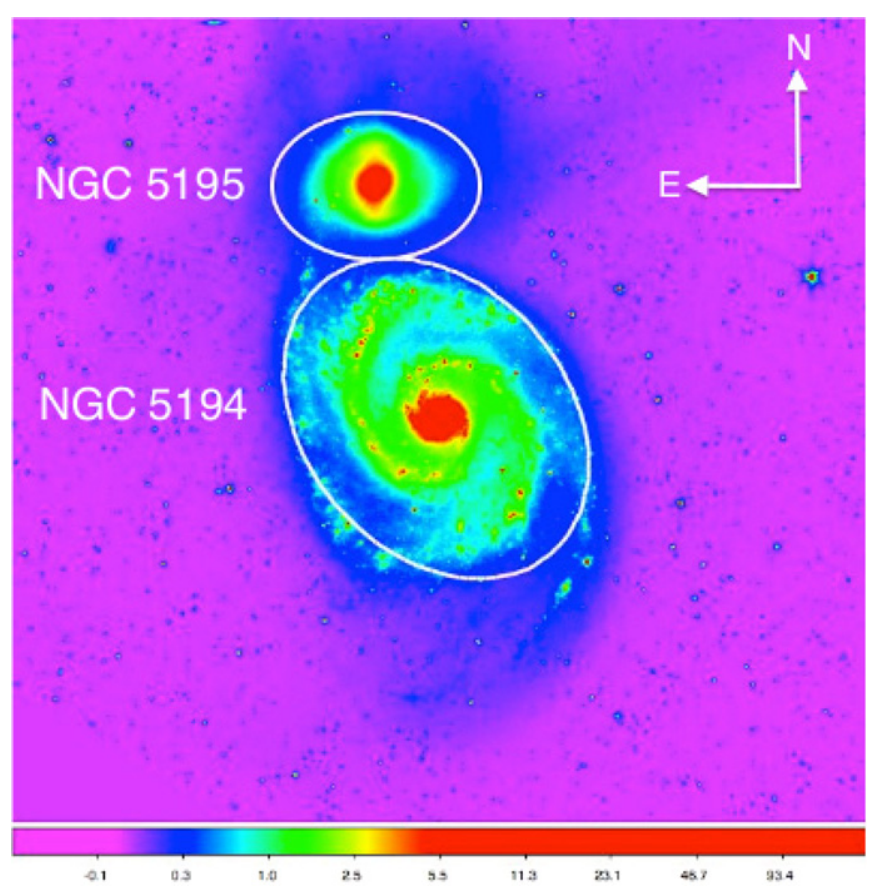

Figure 1. Image of the Whirlpool galaxy system, NGC 5194/5 (M51a/b) from the Spitzer Space Telescope's IRAC camera at $3.6 \mu \mathrm{m}$ (in intensity units of $\mathrm{MJy} \mathrm{sr}^{-1}$ ). Measured apertures are shown for each galaxy and were defined by a constant stellar mass surface density of $50 \mathrm{M}_{\odot} \mathrm{pc}^{-2}$.

(A color version of this figure is available in the online journal.)

surface brightness. We have opted to exclude the $500 \mu \mathrm{m}$ image as a compromise for higher spatial resolution. Boselli et al. (2012) demonstrate that for late-type spiral galaxies the SPIRE FIR colors are tightly correlated and the $500 \mu \mathrm{m}$ band is not necessary to constrain a dust model. In addition, because our analysis hinges on comparing a large number of observations (4000 pixels in 20 images) to large libraries of stellar and dust models, we require satisfactory signal-to-noise $(\mathrm{S} / \mathrm{N})$ levels in each pixel to determine parameter likelihoods. In this section, we describe image processing steps and discuss our efforts to accurately quantify uncertainties in post-processed images.

\subsection{Adopted Galaxy Parameters}

For our analysis we use a common distance of $9.9 \pm 0.7 \mathrm{Mpc}$ (Tikhonov et al. 2009), although a wide range of distances (anywhere from 6 to $10 \mathrm{Mpc}$ ) can be found in the literature (e.g., 8.4 Mpc from Feldmeier et al. 1997 is often used). This latest distance is from Hertsprung-Russell diagram matching using individual red giant stars seen in HST archival images. We also measured our own aperture and galactic center for each galaxy based on constant stellar mass density rather than surface brightness. Our apertures, shown in Figure 1, were defined at a constant stellar mass of $50 M_{\odot} \mathrm{pc}^{-2}$ and ensured that neither galaxy's aperture overlapped. When total mass estimates are measured for each galaxy, it is the sum of pixels within this aperture. No aperture corrections are applied extending to larger galactic radii because beyond our chosen apertures, the galaxies begin to blend. For this reason, mass surface density ratios are more relevant to our analysis. We also use the apertures to define an effective radius, $r_{\text {eff }}$, in order to measure $1 \mathrm{D}$ radial profiles of mass densities and other derived SED properties. Our effective 
Table 1

Structural Properties

\begin{tabular}{lcccccc}
\hline \hline Galaxy & $\begin{array}{c}\text { R.A. (J2000) } \\
(\mathrm{deg})\end{array}$ & $\begin{array}{c}\text { Decl. (J2000) } \\
(\mathrm{deg})\end{array}$ & $\begin{array}{c}2 a \\
(\operatorname{arcsec})\end{array}$ & $\begin{array}{c}2 b \\
(\operatorname{arcsec})\end{array}$ & $\begin{array}{c}\text { P.A. } \\
(\mathrm{deg})\end{array}$ & $\begin{array}{c}\text { Aperture Radius } \\
(\operatorname{arcsec})\end{array}$ \\
\hline NGC 5194 & 202.47065 & +47.19517 & 383 & 286 & 50 & 295 \\
NGC 5195 & 202.49726 & +47.26531 & 225 & 160 & 90 & 115 \\
\hline
\end{tabular}

radius is defined as

$$
\left(\frac{r_{\mathrm{eff}}}{r_{\mathrm{app}}}\right)^{2}=\left(\frac{x}{a}\right)^{2}+\left(\frac{y}{b}\right)^{2},
$$

where $a$ is the semimajor axis, $b$ is the semiminor axis, and $r_{\text {app }}^{2}=a^{2}+b^{2}$. The position angle, P.A., is measured east from north. These parameters are given for reference in Table 1 and shown in Figure 1 imposed on a Spitzer/IRAC $3.6 \mu \mathrm{m}$ image of the galaxies.

\subsection{Far-infrared Data}

The VNGS observed the Whirlpool system with Herschel/ PACS at $70 \mu \mathrm{m}$ and $160 \mu \mathrm{m}$ and with Herschel/SPIRE at $250 \mu \mathrm{m}$ and $350 \mu \mathrm{m}$. Images at each wavelength at their native resolution are shown on the left column of Figure 2. Details on the observational setup can be found in Bendo et al. (2012).

The PACS images processed using both HIPE v5 and SCANAMORPHOS v8. ${ }^{13}$ To account for changes in calibration from HIPE v5 to HIPE v6, the 70 and $160 \mu \mathrm{m}$ images were divided by factors of 1.119 and 1.174. The PACS images were converted to $\mathrm{Jy} \mathrm{sr}^{-1}$ from Jy pixel ${ }^{-1}$ with plate scales of $2^{\prime \prime}$ and $4^{\prime \prime}$ for the $70 \mu \mathrm{m}$ and $160 \mu \mathrm{m}$ images, respectively.

The SPIRE images were processed using HIPE and BRIGADE as described in Smith et al. (2012). The final SPIRE maps were created using the naive mapper provided in the standard pipeline with pixel sizes of $6^{\prime \prime}$ and $8^{\prime \prime}$ at $250 \mu \mathrm{m}$ and $350 \mu \mathrm{m}$. The FWHM of the SPIRE beams for this pixel scale are $18^{\prime \prime} .2$ and $24 . .5$ at $250 \mu \mathrm{m}$ and $350 \mu \mathrm{m}$ (Swinyard et al. 2010). In addition, the $350 \mu \mathrm{m}$ data are multiplied by 1.0067 to update our flux densities to the latest v7 calibration product. The SPIRE images required conversion from $\mathrm{Jy}_{\text {beam }}{ }^{-1}$ to $\mathrm{Jy}^{-1}$ where the beam is the point-spread function (PSF) area size of the instrument at the observed wavelengths taken for this analysis as 423 and $751 \operatorname{arcsec}^{2}$ beam $^{-1}$ for the $250 \mu \mathrm{m}$ and $350 \mu \mathrm{m}$ images, respectively. The SPIRE images were initially in point source relative spectral response function (RSRF) weighted intensities. To convert to extended source RSRF-weighted intensities we divided each image by the $K_{4 p}$ terms given in the SPIRE handbook of $(1.0113,1.0087)$ for the $250 \mu \mathrm{m}$ and $350 \mu \mathrm{m}$ images. The images remained in RSRF-weighted intensities (i.e., not converted to monochromatic intensities or colorcorrected). Model band fluxes are computed by integrating the model spectra with each filter's spectral response function and thus account for the filter's effective wavelength and throughput, as well as the galaxy's varying spectral slope.

The sky background in each image is measured using 10 pixel wide boxes surrounding the system. For all of our Herschel/PACS and Herschel/SPIRE images, we subtracted the median value of the sky region pixels as we found values that were on order $1 \sigma-2 \sigma$ higher (in the case of PACS images) or lower (in the case of our SPIRE images) than the standard deviations in the background pixels. All images are matched to

\footnotetext{
13 http://www2.iap.fr/users/roussel/herschel/
}

a common resolution using kernels created from the PSFs from Aniano et al. (2011). They measured azimuthally averaged empirical PSFs for the Herschel instruments. The images were all convolved to a matched Gaussian PSF (FWHM = 28") slightly higher than the SPIRE $350 \mu \mathrm{m}$ image to ensure the power in the non-Gaussian beam of SPIRE is conserved to the assumed Gaussian beam (Aniano et al. 2011). All images are remapped to a common plate scale of $10^{\prime \prime}$ pixel $^{-1}$ using the WCS tools program REMAP. ${ }^{14}$ The resulting processed images are shown in the middle panel of Figure 2.

\subsection{Optical and Near/Mid-infrared Data}

Our optical ( $B V R I \mathrm{H} \alpha$ ) and mid-infrared (IRAC 3.6, 4.5, 5.6, 8.0, and MIPS $24 \mu \mathrm{m}$ ) observations come from the Spitzer Infrared Nearby Galaxies Survey (SINGS; Kennicutt et al. 2003). Near-infrared $J H K$ imaging is from the 2MASS Large Galaxy Atlas survey (Jarrett et al. 2003). All images are converted to units of $\mathrm{Jy} \mathrm{sr}^{-1}$ and we account for loss of light due to foreground dust attenuation from the Milky Way (MW) using the extinction values provided by $\mathrm{NED}^{15}$ using maps from Schlegel et al. (1998) for all bands up to and including the IRAC $3.6 \mu \mathrm{m}$ image.

We replace emission from foreground stars (in $10^{\prime \prime}$ diameter apertures) with the median value of emission of the pixels contained in its surrounding annulus ( $4^{\prime \prime}$ wide). The stars are identified from the 2MASS Point Source Catalog. In order to ensure we do not remove any $\mathrm{H}$ II regions belonging to the galaxy, we only remove point sources with mid-infrared to optical light ratios comparable to stars, taking the criterion that the $8 \mu \mathrm{m}$ surface brightness cannot be greater than 1.5 times the $R$-band surface brightness. If the ratio is higher, $I_{8} / I_{R}>1.5$, the point source is an $\mathrm{HI}$ region (identified by its bright $\mathrm{PAH}$ emission at $8 \mu \mathrm{m}$ ) within the galaxy and is not removed.

Also, we correct for any constant in the sky background in each image by placing 10 boxes, each 10 pixels wide, throughout the sky in each image. If the median in this background value is larger than the standard deviation across the boxes, we subtracted this constant from the image. The narrowband $\mathrm{H} \alpha$ image (not continuum subtracted) was the only image that required this step. Finally, we convolve the images with appropriate kernels to our chosen common resolution of $28^{\prime \prime}$ assuming Gaussian PSFs for the optical and near-infrared images. The FWHMs of the PSFs for the optical images are 2 ." $0,1.9,1$." $6,1^{\prime \prime} .9$, and $1^{\prime \prime} .8$ for the $B, V, \mathrm{H} \alpha, R$, and $I$ bands, respectively. For the NIR images, and the FWHM of the PSFs are $3 . .3,3$.'2, and $3^{\prime \prime} .5$ for the $J, H$, and $K$ bands.

The Spitzer images are convolved to a $28^{\prime \prime}$ Gaussian using kernels from Aniano et al. (2011). The FWHMs of the Spitzer images are 1".90, 1".81, 2".11,2".82, and 6."43 for the IRAC $3.6 \mu \mathrm{m}, 4.5 \mu \mathrm{m}, 5.6 \mu \mathrm{m}, 8.0 \mu \mathrm{m}$, and MIPS $24 \mu \mathrm{m}$ images, respectively. All images are remapped to a common plate scale

\footnotetext{
14 http://tdc-www.cfa.harvard.edu/wcstools

15 http://ned.ipac.caltech.edu/
} 

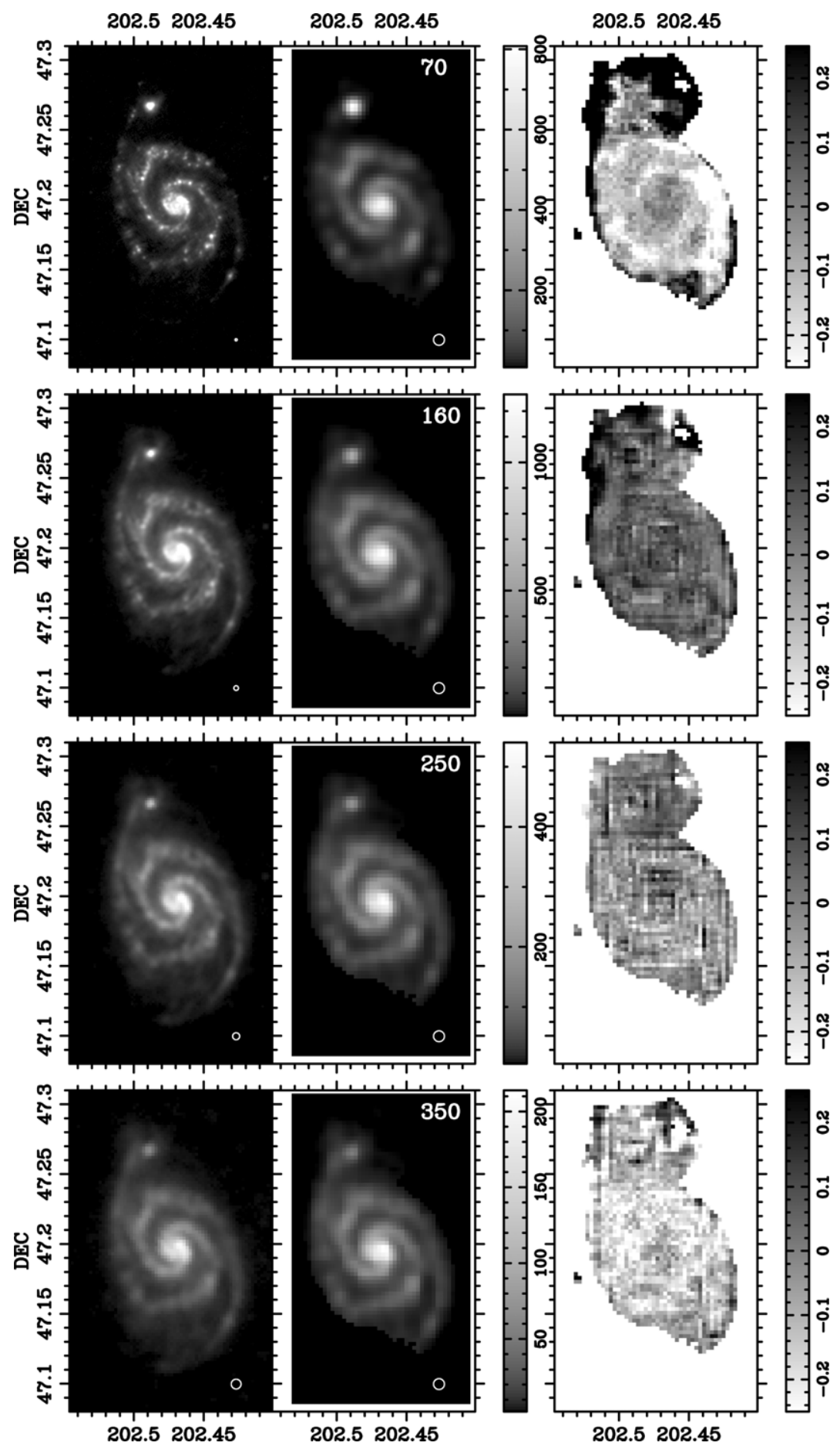

Figure 2. Far-infrared images of NGC 5194/5 from Herschel/PACS and SPIRE as part of the VNGS survey from top to bottom at $70 \mu \mathrm{m}, 160 \mu \mathrm{m}, 250 \mu \mathrm{m}$, and $350 \mu \mathrm{m}$. The left panel is the original image, while the middle panel shows the image after matching it to a common resolution of $28^{\prime \prime}$ and plate scale of $10^{\prime \prime}$ pixel ${ }^{-1}$ Both images are shown in $\mathrm{MJy} \mathrm{sr}^{-1}$ and the beam area for each image is shown in the bottom right. The right shows the residual image between the models and observations, normalized by the observations: $\left(I_{\lambda, \text { obs }}-I_{\lambda, \text { model }}\right) / I_{\lambda \text {,obs. }}$. The best-fit model images from Draine $\& \operatorname{Li}(2007)$ match the images at $160 \mu \mathrm{m}$ and $250 \mu \mathrm{m}$ to within $\sim 10 \%$, but underestimate emission in some regions of the $70 \mu \mathrm{m}$ (top) and $350 \mu \mathrm{m}$ (bottom) images by over $20 \%$.

of $10^{\prime \prime}$ pixel $^{-1}$ using the WCS tools program remap. ${ }^{16}$ For reference, we have included images of the galaxy in each band, before and after image processing in the Appendix of this paper.

\subsection{Neutral and Molecular Gas}

Imaging of the neutral gas is provided by The HI Nearby Galaxy Survey (THINGS; Walter et al. 2008). Already in units

\footnotetext{
16 http://tdc-www.harvard.edu/wcstools
}

of $M_{\odot} \mathrm{pc}^{-2}$, we match plate scales and convolve the beam assumed to be a Gaussian of FWHM $=6^{\prime \prime}$ to a $28^{\prime \prime}$ beam to match our other observations. Molecular gas observations at $\mathrm{CO}(1-0)$ from the Nobeyama $45 \mathrm{~m}$ telescope (Koda et al. 2011) are also included in the analysis. The $\mathrm{CO}(1-0)$ image, originally in antenna temperature units of $\mathrm{K} \mathrm{km} \mathrm{s}^{-1}$, is divided by 0.4 to convert to main beam temperature units. We then use a constant conversion factor of $X_{\mathrm{CO}}=2.0 \times 10^{20} \mathrm{~mol} \mathrm{~cm}^{-2}$ $\left(\mathrm{K} \mathrm{km} \mathrm{s}^{-1}\right)^{-1}$ (Strong et al. 1988), which corresponds to 

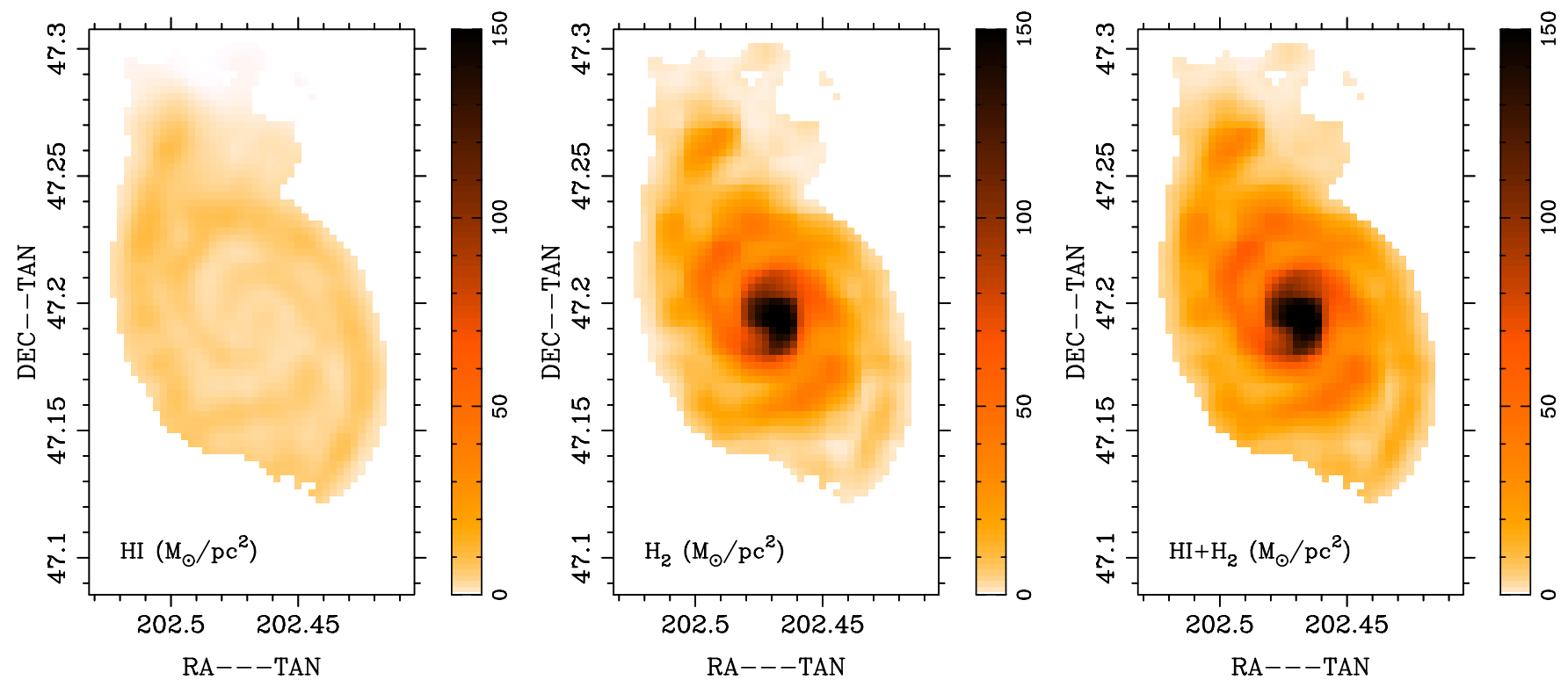

Figure 3. Left: H I mass surface density image of NGC 5194/5 from the THINGS survey (Walter et al. 2008) convolved to match the PSF (FHWM $=28^{\prime \prime}$ ) of our other observations. Middle: the $\mathrm{CO}(1-0)$ image from the Nobeyama $45 \mathrm{~m}$ telescope (Koda et al. 2011) converted to an $\mathrm{H}_{2}$ mass surface density image. Right: total gas mass surface density image is created by summing the two left images. All images are in units of $M_{\odot} \mathrm{pc}^{-2}$ and are shown on the same intensity scale. Molecular gas dominates the gas emission in the central area of the galaxy and along the spiral arms. To convert to total gas mass surface densities, a 1.36 scaling is required to account for helium.

(A color version of this figure is available in the online journal.)

$3.2 M_{\odot} \mathrm{pc}^{-2}\left(\mathrm{~K} \mathrm{~km} \mathrm{~s}^{-1}\right)^{-1}$ (Wilson et al. 2009), to convert the $\mathrm{CO}$ intensities to an $\mathrm{H}_{2}$ gas mass surface density. This image is then convolved from a $22^{\prime \prime}$ beam to a $28^{\prime \prime}$ beam to match all other observations as well as changing the plate scale to $10^{\prime \prime}$ pixel $^{-1}$. Both images of the gas mass surface densities are shown in Figure 3. The sum of both the neutral and molecular gas is shown on the right. All images are multiplied by 1.36 to account for helium and thus represent the total, not just hydrogen, gas in the galaxy. We use the total gas mass surface density in our comparisons to the stellar and dust mass surface densities.

\subsection{Uncertainties and Qualifying Signal-to-noise Criteria}

We generally considered two types of uncertainties in each pixel value of each image: the error due to the uncertainty in the sky background and also the calibration uncertainty. The uncertainty in the sky background is measured in each image after background subtraction and convolving to lower spatial resolution by selecting ten $100^{\prime \prime}$ wide boxes throughout the image. The standard deviation in the median value of the pixels in each box leads to our uncertainty in the sky background. Calibration uncertainty is taken from the various instrumental handbooks or survey description papers. Calibration uncertainties in the optical data are assumed to be $5 \%$, except for a $10 \%$ uncertainty in the narrowband $\mathrm{H} \alpha$ image. Both 2MASS and Spitzer images have calibration uncertainties on the order of $3 \%$. Uncertainties for Herschel observations are $3 \%$ and $5 \%$ at $70 \mu \mathrm{m}$ and $160 \mu \mathrm{m}$, respectively and $7 \%$ at both $250 \mu \mathrm{m}$ and $350 \mu \mathrm{m}$. In addition, error maps of the Herschel images were also included in the analysis. These images were processed to a common spatial scale as the images above by taking the square root of the convolution of the square of the error image with the square of the kernel. Furthermore, the error images were matched to the $10^{\prime \prime}$ pixel $^{-1}$ plate scale. Uncertainties in each pixel were added in quadrature to produce a combined error map at each bandpass.

We want to include as many pixels in our analysis as possible and at the same time exclude regions in which the majority of pixels have low $\mathrm{S} / \mathrm{N}$ levels. A fit to the SED of a pixel was performed if at least five bands had $\mathrm{S} / \mathrm{N}>3$ in the optical/near-infrared data from $B$ through $K$ bands. We also required $\mathrm{S} / \mathrm{N}>3$ in at least three bands for the infrared data from $5.6 \mu \mathrm{m}$ to $350 \mu \mathrm{m}$. In practice, this selection was dominated by the relatively less sensitive infrared data; every pixel which satisfied the infrared criteria had $\mathrm{S} / \mathrm{N}>3$ in all optical bands.

\section{SED LIBRARIES AND FITTING RESULTS}

Although models exist (e.g., Devriendt et al. 1999; da Cunha et al. 2008; Noll et al. 2009; Popescu et al. 2011) that describe both stellar and dust emission together, they tend to be either computationally expensive (requiring many fitted parameters and slow codes computing the uncertainties in these parameters) or lack the most up-to-date SEDs. Unlike today's common stellar population synthesis (SPS) codes-PEGASE.2 (Fioc \& RoccaVolmerange 1997), GALEXEV (Bruzual \& Charlot 2003), and FSPS (Conroy et al. 2009) - these self-consistent stellar and dust emission codes are not extensively tested and their use is not widespread. For this reason, we opt to fit the stellar SED and dust SED components separately. This approach is similar to that of Noll et al. (2009), but by considering both components separately we can fit the SED at a much faster speed; this is primarily out of necessity since we are dealing with a very large data set (20 images with 4000 pixels each). The downsides, of this approach are first that energy is not necessarily conserved from the optical to IR (such that the re-processed infrared emission is linked to the ionizing radiation provided by the stellar populations), something that both MAGPHYS (da Cunha et al. 2008) and CIGALE (Noll et al. 2009) do achieve, and second the near- to mid-infrared regime from $\sim 1-5 \mu \mathrm{m}$, where both dust and stellar emission are significant, cannot be used to constrain the fit. However, because both pre-main-sequence and post-main-sequence stellar emission are not well calibrated in 
the SPS models at these wavelengths (nor is PAH emission) it is not ideal to fit this regime in any case.

In our method, we first fit our $B$ - through $K$-band photometry to stellar emission models using standard SPS codes as we describe in Section 3.1. Using the predicted stellar emission from these fits, we are able to subtract stellar emission from the mid-infrared images at $5.6 \mu \mathrm{m}$ and $8.0 \mu \mathrm{m}$. As we will describe in Section 3.2, we then fit the 5.6 through $350 \mu \mathrm{m}$ images to the mid- through far-infrared dust emission models from Draine \& Li (2007), as well as fitting a single temperature modified blackbody model.

\subsection{Stellar SEDs}

A number of codes exist for a user to generate a model galaxy SED. The choice of parameters and assumptions that are entered into the codes can lead to systematic differences in the output parameters. Stellar masses are most robust and can be predicted to within 0.2 dex (Muzzin et al. 2009; Conroy et al. 2010) and often to higher precision if near-infrared photometric bands are included, although masses will vary systematically depending on the choice of IMF. Other parameters tend to have larger dispersions across different codes (Muzzin et al. 2009). If uncertainties in the inputs (such as evolutionary isochrones, stellar libraries, binary fraction) of the code are considered, then masses are only good to within $\sim 0.3$ dex (Conroy et al. 2010). For a more intensive discussion on systematic differences and known uncertainties of SPS models, the reader is referred to papers by Muzzin et al. (2009) and Conroy et al. (2009, 2010).

In this study we take a more empirical approach and try to compare the best-fit model SED parameters to information from other observations and independent studies. For example, we have some prior knowledge regarding the star-forming history of the system based on isochrones (which have some of the same uncertainties as those found in SPS codes) fit to individuals stars, most recently from Tikhonov et al. (2009). They indicate an enhancement in star formation around $400 \mathrm{Myr}$ ago in both galaxies. Independently, previous dynamical studies of the system provide a consistent temporal view of the system. The motions of gas and stars in the galaxies suggest at least one encounter between the two galaxies 300-500 Myr ago from two independent studies from Salo \& Laurikainen (2000) and Dobbs et al. (2010). This prior knowledge of the star formation history (SFH) could be used to constrain our fitting, but instead we have opted to keep the SFH, stellar population ages, and dust attenuation as free parameters to see how they compare to these independent studies.

Our stellar libraries are generated by the extensively tested PEGASE.2 (Fioc \& Rocca-Volmerange 1997) code and are an extension of work done in Glazebrook et al. (2004) and Mentuch et al. (2009) and include four metallicities: $Z=$ $\{0.004,0.008,0.02,0.05\}$. We assume no evolution in the metallicity and no gas infall, although this is likely not the best explanation for NGC 5195 in particular since it appears to be accreting material from NGC 5194. Our adopted IMF is that of Kroupa (2001). The SFH is modeled as a sum of a continuous star-forming population (CSP) described by a tauexponential and an additional burst of star formation whose amplitude and age are both free parameters. The SFH of the continuous population is modeled as an exponentially decreasing function of time $\left(\exp ^{-t / \tau}\right)$, parameterized by an $e$-folding timescale of $\tau=\{100,500,1000,5000,500000\}$ Myr where $100 \mathrm{Myr}$ is a short burst of star formation or roughly an SSP and the largest timescale of 500 Gyr represents a constant SFH. The burst component is modeled as a tau-exponential with $\tau=100$ Myr. The time since the star formation burst is allowed to be an additional parameter and was given the largest range and sampling at steps of $50 \mathrm{Myr}$ from 50 to $1000 \mathrm{Myr}$, then steps of $250 \mathrm{Myr}$ from $1 \mathrm{Gyr}$ to $5 \mathrm{Gyr}$ and then steps of $500 \mathrm{Myr}$ from $5 \mathrm{Gyr}$ to $13 \mathrm{Gyr}$. The age of the galaxy could be anywhere between $1 \mathrm{Gyr}$ and $13 \mathrm{Gyr}$ with fairly high temporal sampling as well. The mass of the burst compared with the mass of the CSP ranged as $\log \left(M_{\text {burst }} / M_{\mathrm{CSP}}\right)=$ $\{-3,-2,-1,-0.75,-0.5,-0.25,-0.1,0,0.1\}$. Thus, the burst SSP could be anywhere from 0.001 to 1.25 times the mass of the primary CSP.

As we did not include UV observations in this analysis, the nuances of the $2175 \AA$ bump did not have to be considered in the choice of a dust attenuation law. We opted to use the MW dust attenuation curve from Pei (1992) and only considered a screen type geometry of the dust. This law, compared with those of Calzetti et al. (1994) and Charlot \& Fall (2000), for example, more accurately describes the dust attenuation of stars in the MW (Indebetouw et al. 2005) observed in the near- and midinfrared (Mentuch et al. 2010). Curves that represent starburst galaxies (Calzetti et al. 1994; Charlot \& Fall 2000) incorrectly model the dust attenuation because they have not accounted for non-stellar emission that is quite large in star-forming regions (Mentuch et al. 2010), which becomes significant at wavelengths beyond $\sim 1 \mu \mathrm{m}$ (Mentuch et al. 2009). The amplitude of the dust attenuation was an additional free parameter ranging from $A_{V}=0-2 \mathrm{mag}$ in steps of $0.25 \mathrm{mag}$.

We place the modeled galaxies at a distance of $9.9 \mathrm{Mpc}$ to match the system's distance and integrate the synthetic SEDs across each observed band's filter response curve to get theoretical band fluxes. A least-squares comparison between observed and modeled band fluxes in each pixel selects the bestfit model from our suite of stellar models to our BVIRJHK maps. The stellar mass in each pixel is inferred from the least-squares normalization of the model to observed band fluxes.

Following standard Monte Carlo error simulations, our errors are derived for individual parameters by running our fitting routine 100 times, each time allowing the pixel values in each band to sample the Gaussian probability distribution of each measurement. The uncertainty, or $1 \sigma$ spread in the Gaussian distribution, accounts for calibration uncertainty, sky background limitations, and other uncertainties as described in Section 2.5. The mean and standard deviation of the best-fit parameters from all trials lead to our best estimate parameter value and its uncertainty in each pixel.

Figure 4 shows four examples of fits to individual pixels in different regions of NGC 5194/5. The purple curve shows the best-fit SED to the $B-K$ pixels. The large decrease in amplitude from the nuclear region of NGC 5194 (top left) to the arm regions (top right and bottom left) is due to a decrease in stellar mass density. Overall we find that the models fit the observations to better than $10 \%$ and are consistent with the observations within our estimated uncertainties. In the Appendix, we show quality of fit images for the optical, near-infrared, and midinfrared images. Consistently we have very good fits of the stellar emission, with $\chi_{\text {reduced }}^{2} \sim 1$ for most of NGC 5195 and $\chi_{\text {reduced }}^{2} \sim 0.5-1$ for NGC 5194. Only the H $\alpha$ image (which we do not use to constrain the SPS model) is slightly mismatched. We have simplified the SFH in the model to consist of just two components. But we know that for NGC 5194, it consists of at least two continually star-forming episodes: one old stellar 

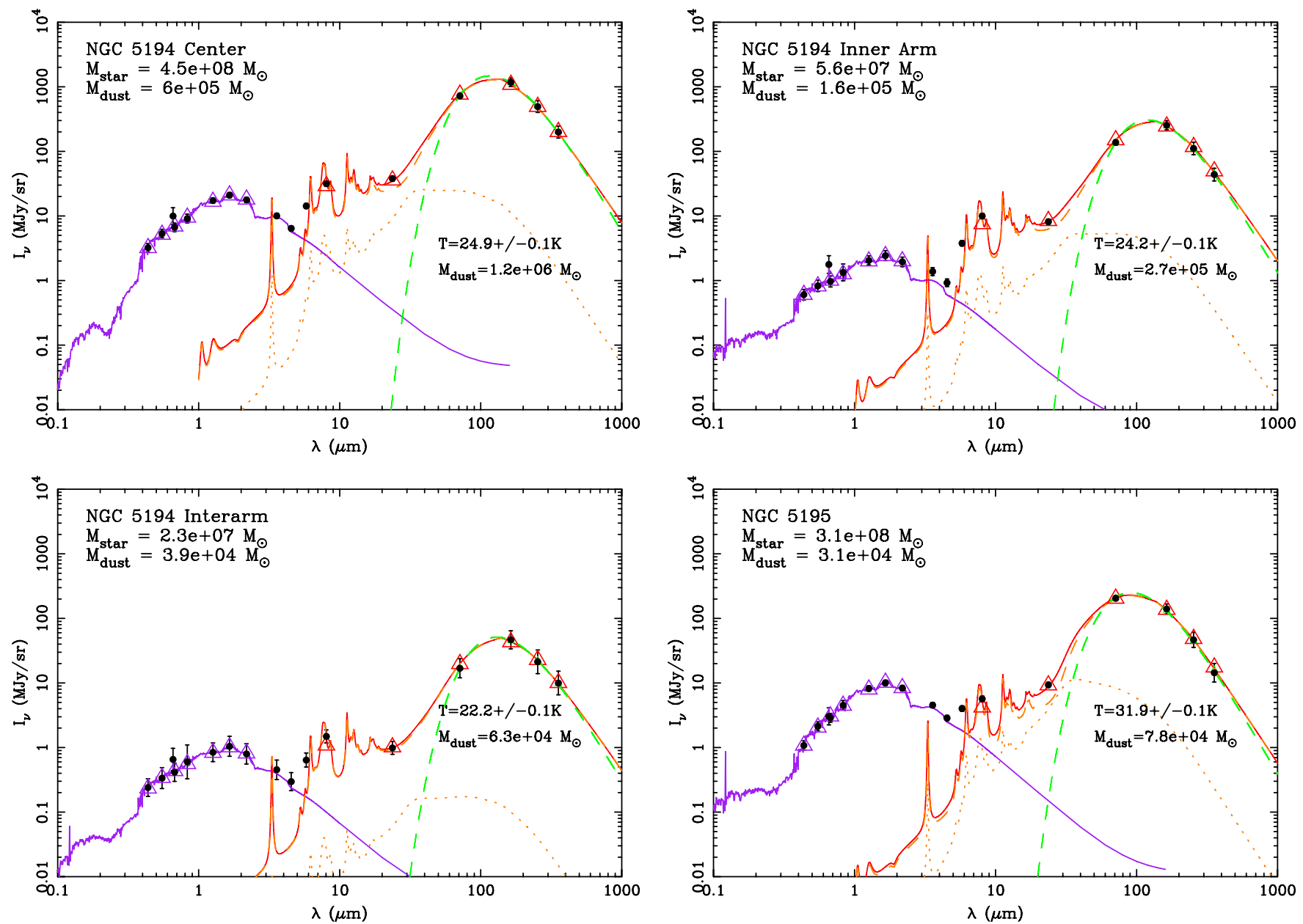

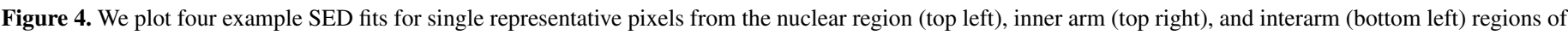

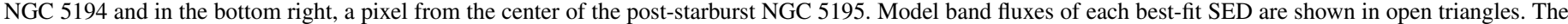

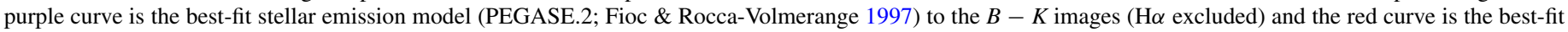

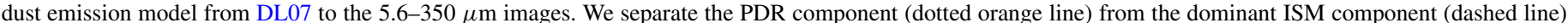

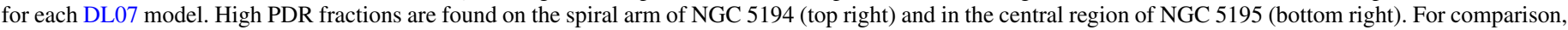

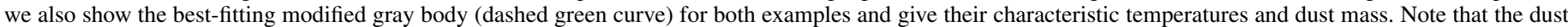

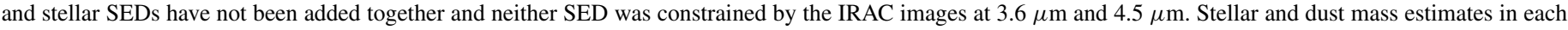

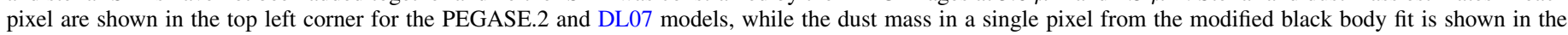
bottom right corner.

(A color version of this figure is available in the online journal.)

population and then one that has been forming stars since at least its encounter with NGC 5195 and likely earlier given its gas fraction and bulge-disk ratio. But our simplified SFH only consists of one continually star-forming population and then a burst population. Because the current star formation is not being accounted for properly, neither is its associated emission in the $\mathrm{H} \alpha$ narrowband image.

Maps of the stellar mass surface density and fractional uncertainties $\left(M_{\star, \text { rms }} / M_{\star, \text { avg }}\right)$ are shown in Figure 5. On average, stellar masses in each pixel can be found to a precision of $0.1 \mathrm{dex}$ based on our Monte Carlo simulations. The distribution of stellar mass radially decreases from the nuclear regions of both galaxies and there is a slight enhancement of stellar mass along the spiral arms in NGC 5194.

Star formation history parameters. In Figure 6 we give radial plots of parameters describing the SFH of the system. The profiles go out to a radial distance of $295^{\prime \prime}$ for NGC 5194 and $115^{\prime \prime}$ for NGC 5195. Beyond these radii the two systems significantly overlap. The left panel shows the age of the old stellar population. There is no significant radial dependence and both systems have similar old stellar population ages of $\log t_{\star}[\mathrm{yr}]=9.9 \pm 0.2$. Thus, the age is not well constrained for the system, ranging from $\sim 7-10 \mathrm{Gyr}$. Somewhat reassuringly, the SED fits reveal, as we show in the middle panel of Figure 6, that there was an increased episode of star formation across the system. For the inner radius of $295^{\prime \prime}$ of NGC 5194 we find a burst occurred $(3.8 \pm 0.9) \times 10^{8} \mathrm{yr}$ ago and for the inner $115^{\prime \prime}$ of NGC 5195 the average time since the burst is $(4.2 \pm 0.8) \times 10^{8} \mathrm{yr}$ ago, consistent with the dynamical models of Salo \& Laurikainen (2000) and Dobbs et al. (2010), and the color-magnitude diagrams of individual red giant branch stars seen by HST (Tikhonov et al. 2009). Our analysis suggests the fraction of mass, relative to the primary population, formed in the burst in NGC 5194 is $0.12 \pm 0.05$ and in NGC 5195 is $0.10 \pm 0.05$. A radial profile of the burst fraction for both galaxies is shown in the right panel of Figure 6 and again, no spatial dependence is found.

Metallicity. While the SFH parameters are generally well mixed in the galaxy at the spatial resolution of this work $(\sim 1 \mathrm{kpc})$, the metallicity of the stars shows a trend of decreasing metallicity 

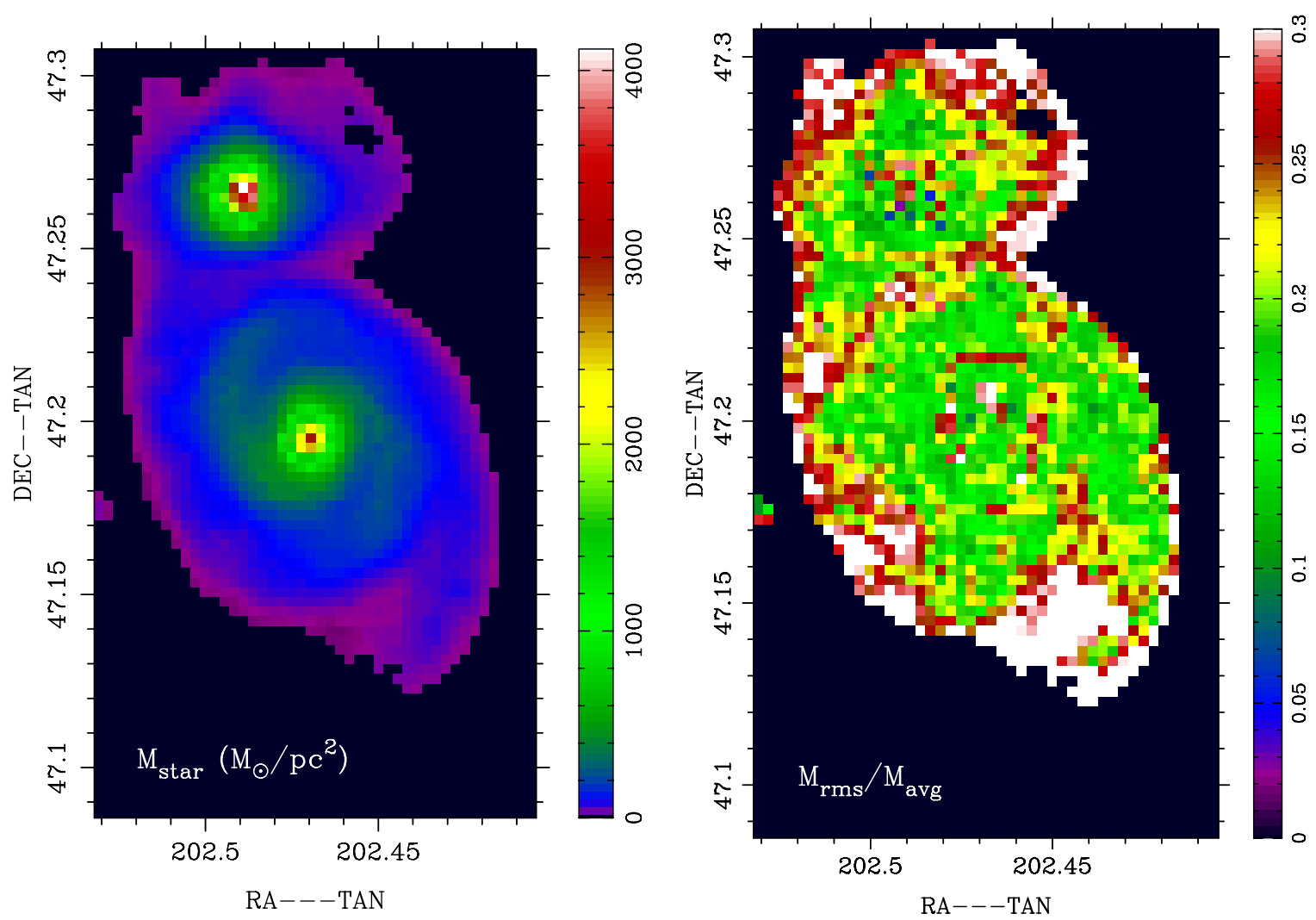

Figure 5. Stellar mass surface density in $M_{\odot} \mathrm{pc}^{-2}$ is shown in the left panel and the normalized uncertainty in the pixel mass determination is shown in the right panel as $M_{\mathrm{rms}} / M_{\text {avg }}$.

(A color version of this figure is available in the online journal.)
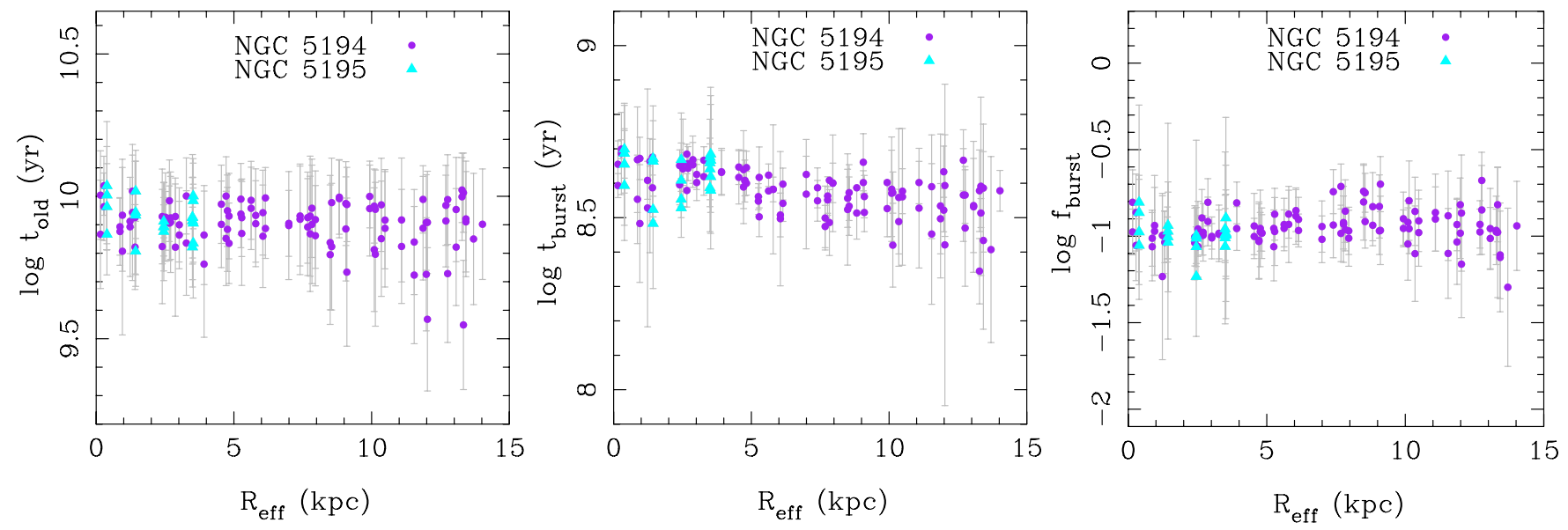

Figure 6. Radial profiles of SED-derived star formation history parameters for NGC 5194 in purple dots and NGC 5195 in cyan triangles. Left: the age of the old stellar population is $\log t_{\star}=9.9 \pm 0.2$. Middle: age of the burst component. A secondary star formation burst period happened 300-600 Myr ago. Right: the mass fraction of the secondary burst component relative to the older primary stellar population is $\sim 5 \%-15 \%$ throughout the galaxy. Note that the deviation from pixel to pixel tends to be less than the uncertainty in the parameters indicating that at the spatial scale of our analysis $\left(28^{\prime \prime}\right)$, the stars are well mixed.

(A color version of this figure is available in the online journal.)

values with radius. In Figure 7, we show a map of the metallicity, where the metallicity transitions from being super solar in the nuclear regions of both galaxies (topping out at $Z=0.05$, our maximum fitted metallicity value) to being near solar $(Z=0.02)$ on the outer galaxy edges. The uncertainty from our Monte Carlo simulations is quite large because of the poor sampling of this parameter available by PEGASE.2.

The right plot shows the 1D radial profile of the metallicity for NGC 5194. Our results are consistent, but not as precise, with radial metallicity profiles from Bresolin et al. (2004) and Moustakas et al. (2010), who also find that metallicity decreases with radius in NGC 5194. Most late-type galaxies exhibit decreasing metallicity gradients (Moustakas et al. 2010), whose slopes depend on the calibration method to convert line strengths to abundances and differ from galaxy to galaxy. Both Bresolin et al. (2004) and Moustakas et al. (2010) measured the metallicity radial profile in NGC 5194 using abundances derived from optical spectroscopy of $\mathrm{H}$ II regions. We convert the best-fit 

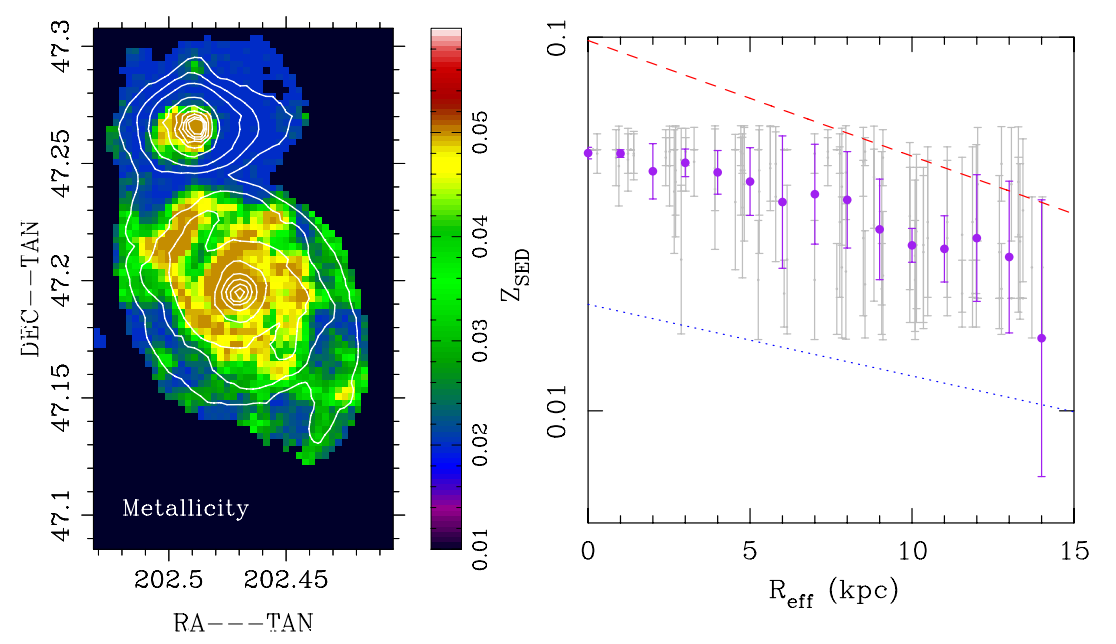

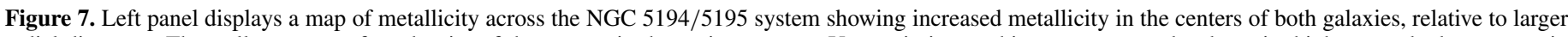

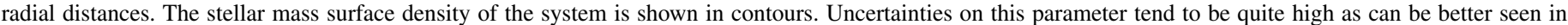

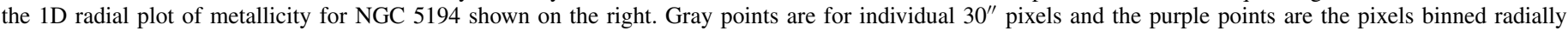

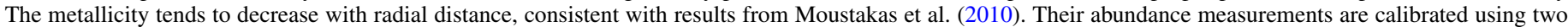

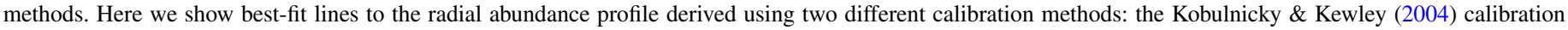
method in dashed red and the Pilyugin \& Thuan (2005) calibration method in dotted blue.

(A color version of this figure is available in the online journal.)

abundance profiles from Moustakas et al. (2010) to a metallicity radial profile (assuming $Z_{\odot}=0.02$ ) using the value for solar metallicity $\left(12+\log (\mathrm{O} / \mathrm{H})_{\odot}=8.69 \pm 0.05\right)$ from Asplund et al. (2009). Moustakas et al. (2010) use two calibration methods to convert line strength to chemical abundances. We plot both calibration methods in Figure 7. The red dashed line has been converted following the Kobulnicky \& Kewley (2004) calibration method and the blue dotted line following the Pilyugin \& Thuan (2005) calibration method. Our values lie somewhere in the middle, but the same trend in decreasing metallicity is seen. Systematic offsets result from our assumed solar metallicity as well as the calibration method. The reader is referred to Moustakas et al. (2010) for more precise metallicity results.

Dust attenuation. SPS models also include a parameter that quantifies the intrinsic dust attenuation. Figure 8 shows a radial plot of the dust attenuation for each galaxy. There is enhanced dust attenuation in the central regions of both galaxies, which decreases with radius from 0.5 mag of visual dust attenuation in the center down to about $0.2 \mathrm{mag}$. For comparison we plot the radial profile of dust extinction for NGC 5194 measured in the near-UV by Muñoz-Mateos et al. (2009). We convert from $\mathrm{A}(\mathrm{NUV})$ to $\mathrm{A}(\mathrm{V})$ using a factor of three based on the dust extinction models of Pei (1992) and Draine (2003), but caution that this attenuation ratio is sensitive to the UV bump at $2175 \AA$ and can range anywhere from $\sim 2.4$ to 3.3 . The dashed orange lines on Figure 8 demonstrate the systematic uncertainty due to this conversion factor. Error bars show the precision in the measurement from Muñoz-Mateos et al. (2009), which is more precise than ours and generally higher. Dust attenuation is modestly underestimated likely because of the exclusion of $\mathrm{UV}$ - and $U$-band data in our analysis.

\subsection{Dust SEDs}

We use the modeled stellar maps of the galaxy to subtract the contribution of stellar emission from our mid-infrared images of the IRAC $5.6 \mu \mathrm{m}$ and $8.0 \mu \mathrm{m}$ bands. These resulting images, which probe emission related to PAHs, and our other infrared

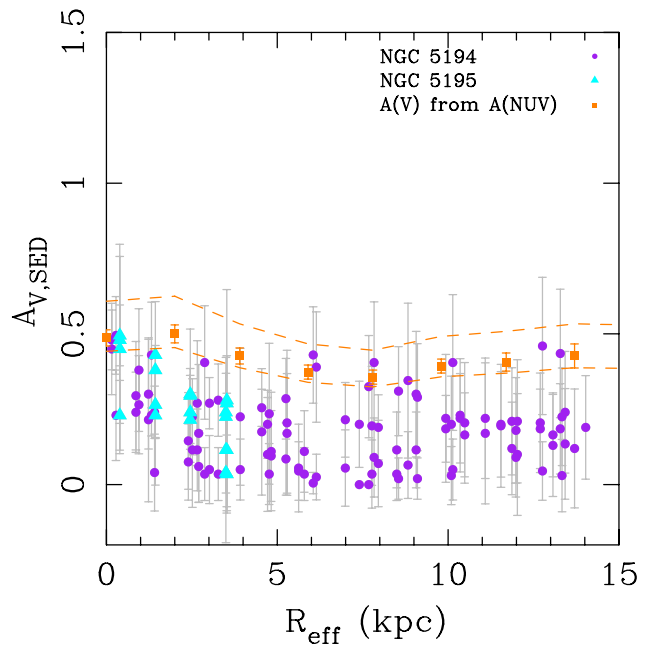

Figure 8. Radial profiles of visible dust attenuation, $A_{\mathrm{v}}$, in units of mags shown for NGC 5194 (purple dots) and NGC 5195 (cyan triangles). $A_{\mathrm{v}}$ is a fitted parameter in our SED stellar population fitting analysis. For comparison, the orange squares show the measured visual extinction from Muñoz-Mateos et al. (2009) inferred from their measured near-UV attenuation. The dashed orange line shows the systematic uncertainty due to the $2175 \AA$ bump.

(A color version of this figure is available in the online journal.)

(24-350 $\mu \mathrm{m})$ images are fit using the two-component emission model put forth by Draine \& Li (2007, hereafter DL07) as well as a conventional single temperature modified blackbody component.

\subsubsection{Draine \& Li (2007) Dust Models}

DL07 model the mid- through far-infrared emission due to PAHs, very small dust grains, and larger dust grains expressed as a function of the underlying ISRF. The dust is assumed to consist of a mixture of carbonaceous grains and amorphous silicate grains, with size distributions that are consistent with the observed wavelength-dependent dust attenuation in the local MW (Weingartner \& Draine 2001), and allows for varying PAH abundances. The infrared emission is a function of the illuminating radiation field, which in this model is assumed to 

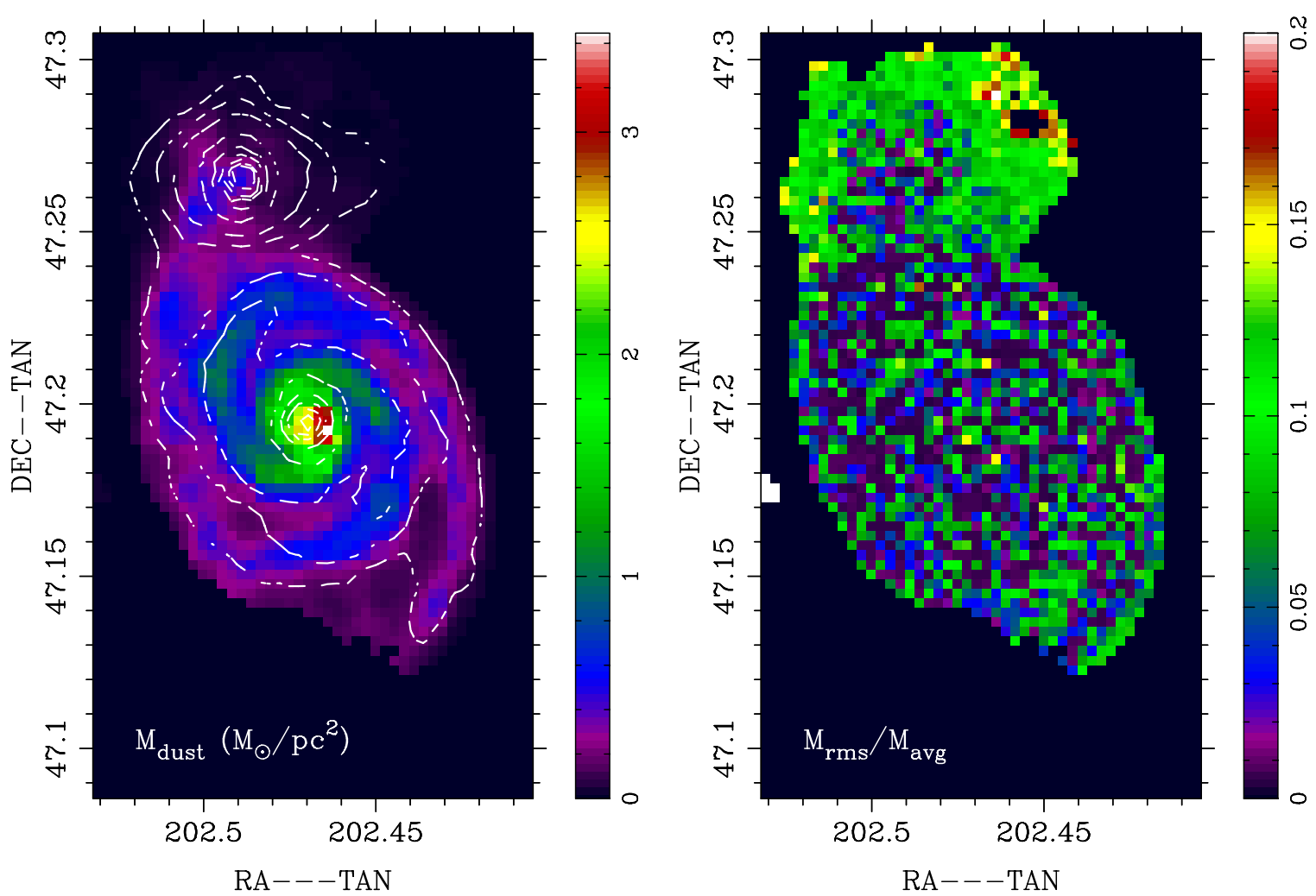

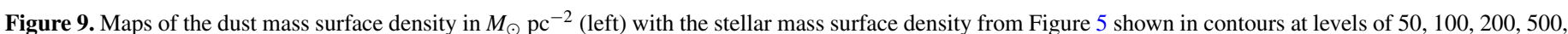

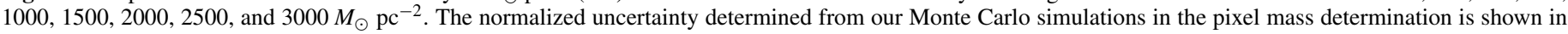
the right panel as $M_{\mathrm{rms}} / M_{\mathrm{avg}}$.

(A color version of this figure is available in the online journal.)

come from two components. Most of the emission comes from diffuse dust heated by the ISRF. The bulk of the dust mass in a galaxy is in this component. A second component represents emission from photodissociation regions (PDRs) where massive star formation creates much stronger radiation fields, leading to relatively higher emission at shorter infrared wavelengths. The diffuse ISRF is scaled to match the spectrum of the ISRF of the MW. The amplitude of the diffuse ISRF is a fitted parameter, $U_{\min }$, normalized to the amplitude of the ISRF of the MW. The radiation field of the PDR component consists of a range of energies from $U_{\min }$ to $U_{\max }$, where $U_{\max }$ can be an additional fitted parameter and $U_{\min }$ is the ISRF of the diffuse component. We opt to fix $U_{\max }=10^{6} U_{\mathrm{Mw}}$ as Draine et al. (2007) showed that it was not a highly sensitive parameter and that most galaxies from the SINGS survey were fit well by this fixed value parameter.

Our model library consists of MW type dust models from DL07 and varies in $U_{\min }$ from $0.1-25 \times U_{\mathrm{Mw}}$. The relative contribution of the secondary PDR component is quantified by the parameter, $\gamma$, and ranges from 0.001 to 1 times the primary interstellar medium (ISM) component. An additional parameter quantifies the mass fractional contribution of PAH emission, $q_{\mathrm{PAH}}$, in the mid-infrared region of the SED with following range of values: $q_{\mathrm{PAH}}=\{0.47,1.12,1.77,2.5,3.19,3.9,4.58\} \%$. Finally, we convert the SEDs from units of per hydrogen nucleon to units per dust mass assuming $M_{\text {dust }} / M_{\mathrm{H}}=0.01$.

As with the stellar SEDs, model band fluxes are generated by integrating the PACS and SPIRE filter response functions over the model SED. The SPIRE filter curves were multiplied by a normalized $\lambda^{2}$ function to convert the response curves from those defined for point sources to those of extended source emission. A least-squares fit is done between the observed bands from $5.6 \mu \mathrm{m}$ to $350 \mu \mathrm{m}$ to obtain the best-fit parameters. Again, average best-fit parameter values and uncertainties are measured from 100 Monte Carlo simulations of the probability distribution of the photometry. The dust mass in each pixel is inferred from the least-squares normalization of the model to observed band fluxes.

Overall, the fits reproduce the 5.6-350 $\mu \mathrm{m}$ emission fairly well, leading to $\chi_{\text {reduced }}^{2}=1-2$ over much of NGC 5195 and the central 200" aperture of NGC 5194. Outside of $r=100^{\prime \prime}$, the quality of fits degrades, resulting in $\chi_{\text {reduced }}^{2}>10$. This can be seen in the disagreement between models and observations at $70 \mu \mathrm{m}$ and $350 \mu \mathrm{m}$ in Figure 2. The right panel shows the quality of fit of the model, defined by the following equation:

$$
\frac{I_{\lambda, \mathrm{obs}}-I_{\lambda, \text { model }}}{I_{\lambda, \mathrm{obs}}} .
$$

In general, we find that the models underestimate the emission at 70 and $350 \mu \mathrm{m}$ by as much as $20 \%$, but at all other wavelengths the photometry and models match to better than $5 \%$ over most of the galaxy.

The dust mass surface density map is shown in Figure 9 along with the fractional uncertainty in deriving the parameter. The dust mass is fairly well constrained by the models, with the parameter having less than $10 \%$ uncertainty over most of the galaxy. The dust mass is better constrained by the dust models than the stellar mass is by SPS models because of the smaller variation in the mass-to-light ratio of dust emission. Unlike the stellar mass map (Figure 5), there is more spatial structure in the dust mass, which is particularly enhanced along the spiral arms, similar to the mass distribution seen in the molecular gas in Figure 3. NGC 5195 does not show a radially decreasing 

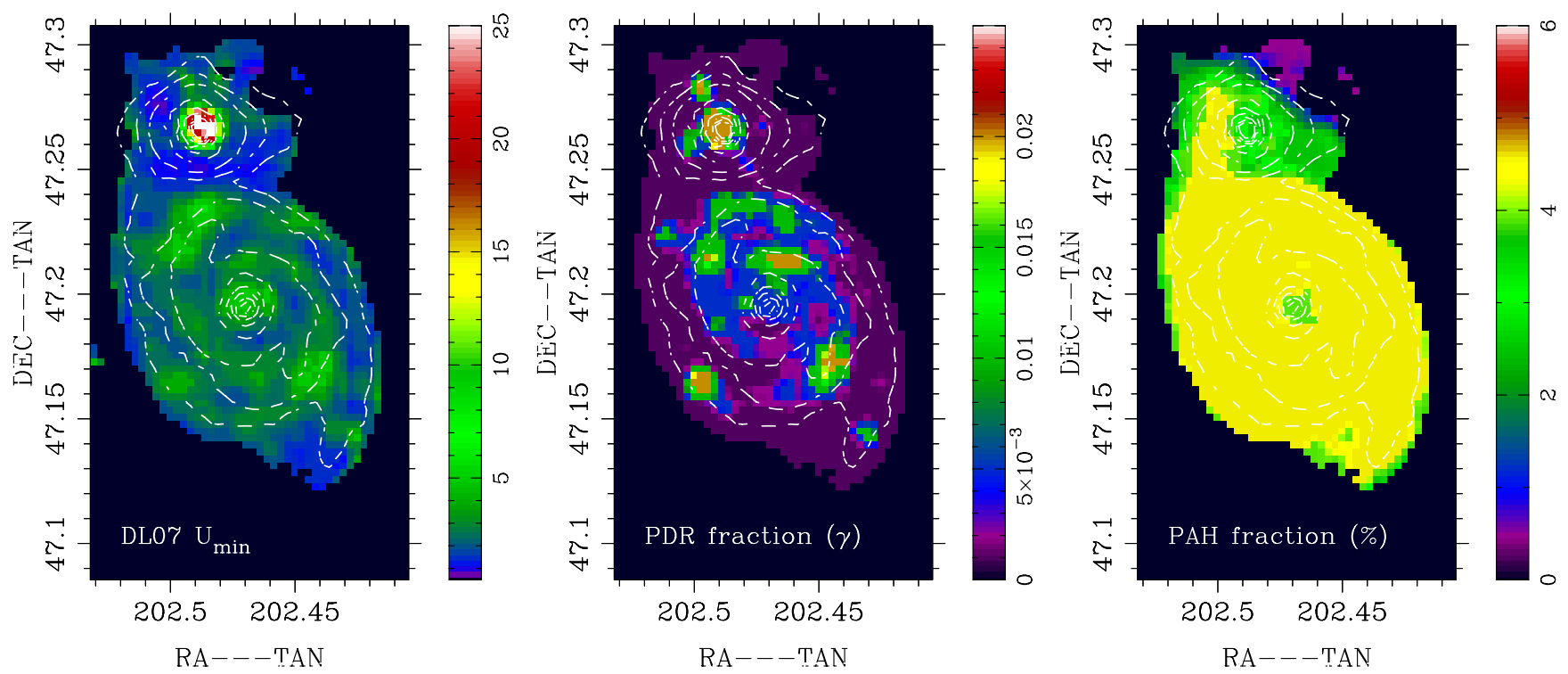

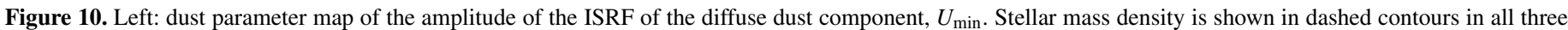

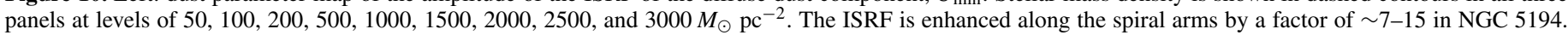

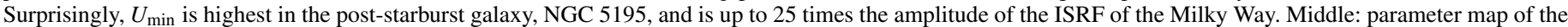

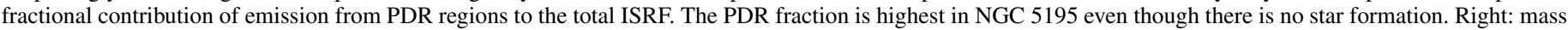

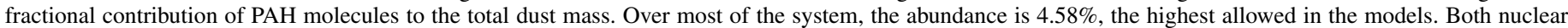
regions show small depressions where both harbor low-luminosity AGNs.

(A color version of this figure is available in the online journal.)

profile as seen in its stellar mass map but still does contain a fair amount of dust in its vicinity and there is some indication of a central concentration. If we consider the higher spatial resolution images from $70 \mu \mathrm{m}$ to $350 \mu \mathrm{m}$ in Figure 2, we observe that the thermal dust emission is very peaked, which could suggest that the dust is concentrated. However, the main reason for this is not that the dust mass is higher, but rather the illuminating radiation is much more intense and concentrated, leading to the peaked infrared emission seen in the infrared images.

The left panel in Figure 10 shows the $U_{\min }$ parameter map of the amplitude of the diffuse ISRF component. The ISRF in NGC 5195 is extremely high, up to 25 times that of the ISRF in the MW. Over most of NGC 5194 the ISRF is very close to the MW value ( $\left.\sim 2-3 U_{\mathrm{MW}}\right)$, but increases by a factor of 7-15 $U_{\mathrm{MW}}$ along the spiral arms. The nuclear region is also higher by a factor of $10 U_{\mathrm{MW}}$. Our formal fitting uncertainties in this map and the one in the middle of the PDR parameter are quite small (about 5\%) indicating the infrared data were quite sensitive to both parameters.

The middle panel shows the fractional contribution of radiation associated with PDR regions, that is, regions with intensities as high as $10^{6}$ times that of the MW. The radiation coming from PDR regions in NGC 5194 is concentrated along the spiral arms but not the nuclear region. About $2 \%$ of the emission in NGC 5195 can be associated with this type of emission, even though there is no massive star formation providing the radiation. The bright emission seen in the infrared images comes from intense radiation fields, not enhanced dust mass. The high dust heating found in NGC 5195 provides strong support for the dust to be located within the galaxy rather than in front of it. But the question then remains as to what is heating the dust? The stellar density is higher in NGC 5195, but not that much higher than in the bulge of NGC 5194, so there is another mechanism at play. We leave this for later discussion in Sections 4.2 and 4.3.

Finally, our SED fits constrain the PAH emission and is shown in the right panel of Figure 10. The PAH fraction is the maximum value allowed by the models of $4.58 \%$ (and thus could feasibly be higher) across most of the system. The fraction remains high along the spiral arms of NGC 5194 extending out to NGC 5195 but then decreases across NGC 5195 to about half the value $\left(q_{\mathrm{PAH}}=3.35 \pm 1.07\right)$ where a compact radio source (van der Hulst et al. 1988) attributed to an AGN exists. There is also a small depression in the abundance in the center of NGC 5194, which is a low-luminosity Seyfert 2 nucleus (Terashima \& Wilson 2001).

\subsubsection{Modified Blackbody Models}

A modified blackbody model was also fit to the 70 though $350 \mu \mathrm{m}$ images as

$$
S_{v}=\frac{\kappa_{v} M_{d} B\left(v, T_{d}\right)}{D^{2}},
$$

where $M_{d}$ is the dust mass with a temperature of $T_{d}$ at a distance, $D$. The emission is expressed as the Planck function, $B\left(v, T_{d}\right)$, modified by $\kappa_{v} \propto v^{\beta}$. Boselli et al. (2012) indicate that for at least late-type, metal-rich galaxies a value of $\beta=2$ appropriately models the SPIRE infrared colors, but caution that $\beta=1.5$ appears to be more appropriate for less metalrich, lower surface brightness late-type galaxies in the Herschel Reference Survey (HRS; Boselli et al. 2010). As a test, we verify that a $\beta=2$ value is sufficient. We performed similar modified blackbody fits allowing $\beta$ to vary from 1 to 3 , but this time fit at lower resolution and include our $500 \mu \mathrm{m}$ SPIRE image. We found that $\beta=2.0 \pm 0.6$ over the whole system.

We normalize for the dust mass at $250 \mu \mathrm{m}$ with $\kappa_{(\lambda=250 \mu \mathrm{m})}=$ $0.398 \mathrm{~m}^{2} \mathrm{~kg}^{-1}$, using the opacity function for the MW from Draine (2003), updated from their dust model presented in Weingartner \& Draine (2001) and consistent with the opacity model from the DL07 models. We assumed a single temperature model, although it should be noted that recent results from Herschel (e.g., Bendo et al. 2010a, 2012; Boquien et al. 2011) 


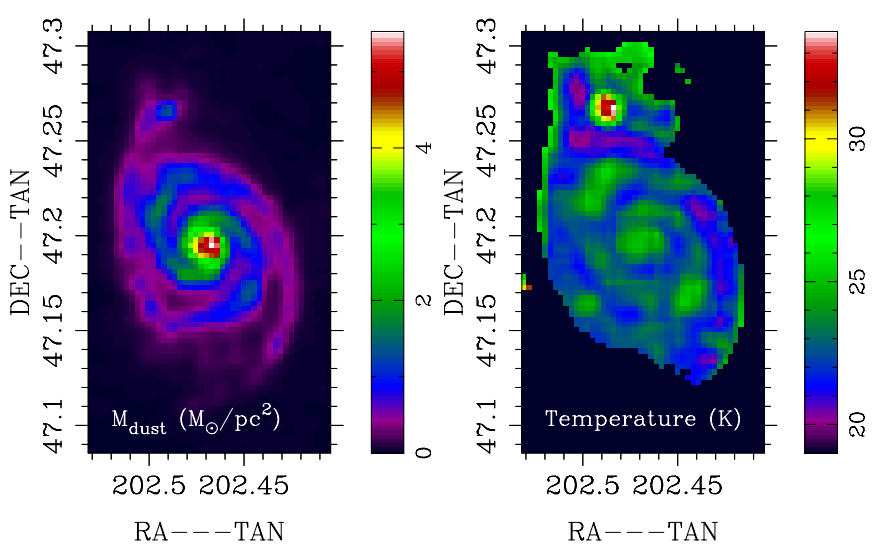

Figure 11. Dust mass surface density (left) and temperature (right) from single temperature modified blackbody models. Just as with the ISRF parameter, $U_{\min }$ from DL07, the dust temperature from modified blackbody fits is largest in the post-starburst galaxy NGC 5195. Temperatures are slightly higher in the nuclear region and along the spiral arms.

(A color version of this figure is available in the online journal.)
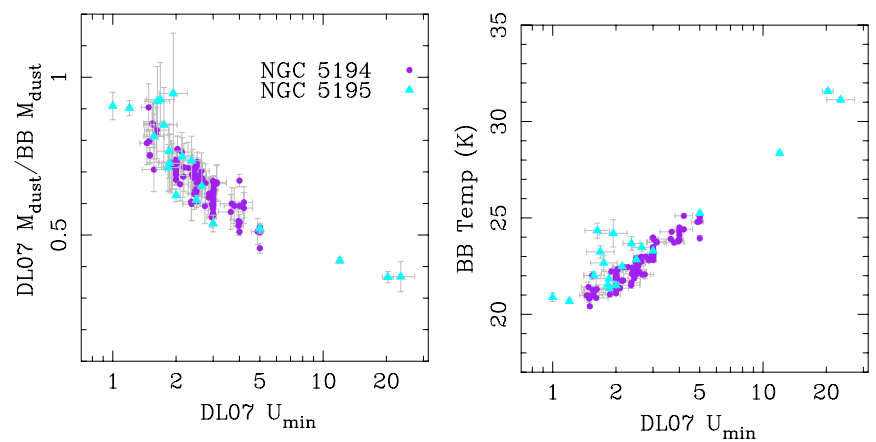

Figure 12. Left: DL07 dust masses relative to single dust temperature dust masses. Right: dust temperature from modified blackbody fits is compared to the DL07 ISRF parameter, $U_{\min }$. As expected, the two parameters are logarithmically related.

(A color version of this figure is available in the online journal.)

suggest such a simplistic model is not appropriate since the dust emitting at $<160 \mu \mathrm{m}$ is not the same thermal component as the dust emitting at $>250 \mu \mathrm{m}$ in nearby spiral galaxies.

As above, band fluxes were generated by integrating the model SED over the filter response curves appropriate for extended source emission. The normalization between the model and observed band fluxes provides the dust mass. Even though our models were normalized at $250 \mu \mathrm{m}$, the fits themselves were not particularly biased toward this band since our leastsquared normalization yields the dust mass rather than normalizing in a single flux band. The infrared observations were sufficiently modeled by the single temperature model resulting in $\chi_{\text {reduced }}^{2} \sim 1$ over the entire system, even though other galaxies are not so easily described. In fact, single temperature models fit all the images from 70 to $350 \mu \mathrm{m}$ as well as the DL07 models if fit to the same band fluxes.

The resulting dust mass distribution, seen in Figure 11, is fairly similar to the one derived using the DL07 models from our Figure 9. The temperature distribution also qualitatively matches the ISRF amplitude map from the DL07 models from our Figure 10. Although the qualitative structure is similar, quantitative pixel-by-pixel comparison of the dust mass shows that systematically modified blackbody masses overestimate the dust mass relative to the DL07 models at high ISRF values. In the left panel of Figure 12, the ratio between derived dust
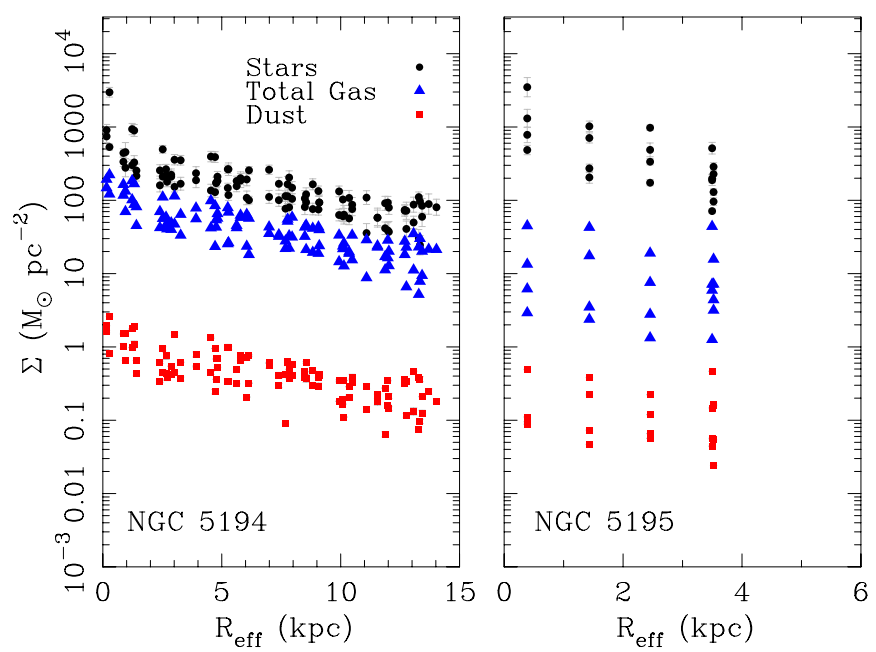

Figure 13. Radial mass profile of the stars (black dots), gas (blue triangles), and dust (red squares) in NGC 5194 (left) and NGC 5195 (right). Each data point represents a $30^{\prime \prime}$ wide pixel box encompassing one FWHM of the convolved beam size. Error bars are smaller than the symbol size if they are not visible.

(A color version of this figure is available in the online journal.)

masses from the DL07 models and modified blackbody models are shown for each galaxy as a function of the amplitude of the ISRF. At higher ISRF, the light-to-mass ratio of the dust is higher. In the central region of NGC 5195 where the ISRF peaks, the dust mass is overestimated by about a factor of three. Without accounting for this variation in the light-tomass ratio, the modified blackbody masses overestimate the dust mass relative to the DL07 dust masses. This is analogous to the optical regime in which young stellar populations exhibit much larger light-to-mass ratios (particularly in the blue) than evolved populations. We also show the relation between dust temperatures and the DL07 $U_{\min }$ from the different models in the right panel. As expected, the two are logarithmically related, although a few values diverge from the trend in NGC 5195.

\section{DISCUSSION}

In the previous section, we presented the output parameter maps resulting from the average best-fit parameters from 100 Monte Carlo simulations of the stellar emission and dust emission fitting routine. Here we compare some of the parameters and discuss their implications. First, we provide mass comparisons and 1D radial profiles of the stellar, gas, and dust mass surface density for both galaxies in Section 4.1. We then turn our discussion to the relation of interstellar heating of the dust in the context of other properties of the galaxies in Section 4.2. We focus on the peculiar properties of the post-starburst NGC 5195 with its high-amplitude ISRF, $\sim 30 \mathrm{~K}$ central dust temperature, and lack of star formation. In Section 4.3, we synthesize its properties and discuss why it is no longer forming stars despite containing molecular gas.

\subsection{Mass Comparisons}

\subsubsection{Radial Projections and Total Masses}

In Figure 13, we plot the radial mass surface density profiles of the stars, gas, and dust in black points, blue triangles, and red squares, respectively, for NGC 5194 in the left panel and NGC 5195 in the right panel. The effective radius, $R_{\text {eff }}$, is determined using the structural parameters listed in Table 1. The apertures of both galaxies were chosen at a constant stellar 
Table 2

Stellar, Gas, and Dust Masses in Each Aperture

\begin{tabular}{lccc}
\hline \hline & $M_{\star}\left(10^{10} M_{\odot}\right)$ & $M_{\text {gas }}\left(10^{10} M_{\odot}\right)$ & $M_{\text {dust }}\left(10^{8} M_{\odot}\right)$ \\
\hline NGC 5194 & $4.7 \pm 0.1$ & $1.10 \pm 0.03$ & $1.19 \pm 0.01$ \\
NGC 5195 & $2.5 \pm 0.2$ & $0.052 \pm 0.005$ & $0.064 \pm 0.001$ \\
Total $^{\mathrm{a}}$ & $7.9 \pm 0.2$ & $1.41 \pm 0.05$ & $1.45 \pm 0.01$ \\
\hline
\end{tabular}

Note. ${ }^{a}$ Total mass in combined system including the mass within both apertures, as well as outside the apertures in the image in which our $\mathrm{S} / \mathrm{N}$ criteria (see Section 2.5) was met.

mass surface density of $50 M_{\odot} \mathrm{pc}^{-2}$. This threshold ensured that the apertures were not overlapping.

In the spiral galaxy, NGC 5194, the profiles of the stars, gas, and dust all show similar slopes, but in NGC 5195, the slope of the stars is slightly steeper and there is no significant slope in the gas and dust distribution. As can be seen in the gas and dust mass surface density plots in Figures 3 and 9, the gas and dust in NGC 5195 appear to be connected to the spiral arm of NGC 5194, rather than centrally concentrated and coupled to the stellar mass in NGC 5195. Both galaxies reach stellar mass surface densities of over $2000 M_{\odot} \mathrm{pc}^{-2}$ in the nuclear regions, then decrease radially to values of $\sim 50 M_{\odot} \mathrm{pc}^{-2}$ at the edge of the apertures (by definition). The central surface density (in a $30^{\prime \prime}$ square region) is only at a value of $3490 \pm 900 M_{\odot} \mathrm{pc}^{-2}$ compared with $2980 \pm 450 M_{\odot} \mathrm{pc}^{-2}$ in NGC 5194.

The dust mass density in the central region of NGC 5194 peaks just above $2 M_{\odot} \mathrm{pc}^{-2}$ in the central region, decreasing to $0.75 M_{\odot} \mathrm{pc}^{-2}$ along the spiral arms and then a constant $0.25 M_{\odot} \mathrm{pc}^{-2}$ throughout the rest of the system. The dust mass density is comparably lower in NGC 5195 at values near $0.25 M_{\odot} \mathrm{pc}^{-2}$, consistent with being an extension of the spiral arm of NGC 5194.

We provide the total stellar, gas, and dust masses in each aperture in Table 2. We also measure the total mass in the combined system, summing up the mass in each pixel over the entire system. A small percentage $(<5 \%)$ of the galaxy is missing from these "total" values as we only added up pixels which satisfied our S/N criteria (see Section 2.5) and low surface brightness regions of the galaxy are not accounted for. About $\sim 80 \%$ of the gas and dust mass is found in the spiral NGC 5194, with the remaining found outside the aperture. Less than $1 \%$ of the gas and 3\% of the dust of the total system are located within the aperture defining NGC 5195. The stellar mass is distributed more evenly between the two galaxies with NGC 5194 being twice as massive as the spheroid NGC 5195. The bulk of the stellar mass is found within our defined apertures due to relatively steeper mass profiles. Only about $10 \%$ of the stellar mass is located outside the two apertures.

\subsubsection{Dust-to-stellar Mass Ratio}

Recall from Figures 5 and 9, which show the stellar and dust mass surface density maps of the system, that the dust mass is slightly more structured than the stellar mass map. In the stellar mass map, there are some enhancements in stellar mass along the spiral arms of NGC 5194, but they are not as pronounced as the spiral arms in the $B$-band image (which can be found in the Appendix), for example, or those found in the dust mass map. Both the $B$-band and dust mass surface density map are enhanced by the younger stellar populations. Because of this, the dust-to-stellar mass ratio, shown in Figure 14, is a factor of two larger along the arms.

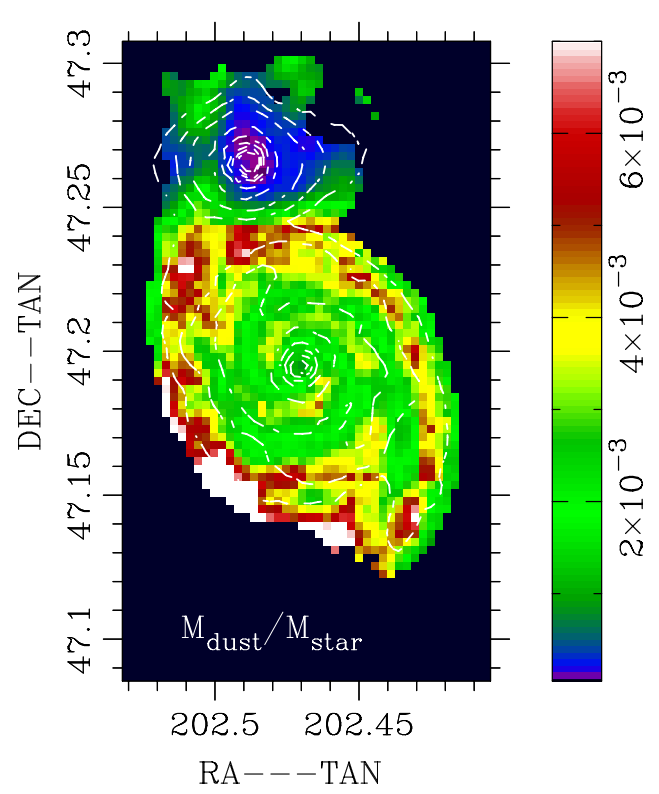

Figure 14. Dust-to-stellar mass ratio map with the stellar mass density given in contours at the levels of 50,100,200, 500, 1000, 1500, 2000, 2500, $3000 M_{\odot} \mathrm{pc}^{-2}$. NGC 5195's dust-to-stellar mass ratio is an order of magnitude smaller than NGC 5194 and increases with radius as the stellar mass decreases. (A color version of this figure is available in the online journal.)

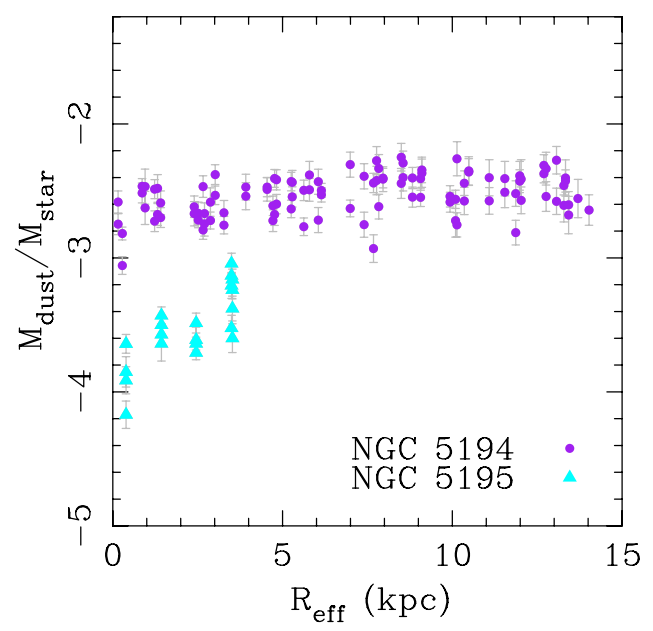

Figure 15. Radial profile of the dust-to-stellar mass ratios in NGC 5194 (purple) and NGC 5195 (cyan). The ratio is relatively constant in NGC 5194 but increases with radius in NGC 5195 primarily due to the decreasing stellar mass density, as demonstrated in Figure 14.

(A color version of this figure is available in the online journal.)

We find an average dust-to-stellar mass ratio of $M_{\text {dust }} / M_{\star}=$ $(3.1 \pm 1.0) \times 10^{-3}\left(\right.$ or $\left.\log \left(M_{\text {dust }} / M_{\star}\right)=-2.5 \pm 0.2\right)$ in NGC 5194. The dust-to-stellar mass ratio is an order of magnitude less in NGC 5195 at $M_{\text {dust }} / M_{\star}=(3.6 \pm 2.2) \times 10^{-4}$ (or $\left.\log \left(M_{\text {dust }} / M_{\star}\right)=-3.5 \pm 0.3\right)$. In Figure 15, the radial profiles of the dust-to-stellar mass ratio are given for both galaxies.

The dust-to-stellar mass ratio of NGC 5194 is consistent with results of other spiral galaxies in the HRS (Boselli et al. 2010 ), which are found to have $\log \left(M_{\text {dust }} / M_{\star}\right)=-2.59 \pm 0.03$ (Cortese et al. 2012). Both galaxies are consistent with the mass ratio found for a large range of galaxy types, $\log \left(M_{\text {dust }} / M_{\star}\right)=$ $-2.95 \pm 0.68$ (Skibba et al. 2011), from the KINGFISH survey (Kennicutt et al. 2011), a Herschel extension of the SINGS survey (Kennicutt et al. 2003). Furthermore, the late-type NGC 5194 is consistent with late-types in KINGFISH, which 

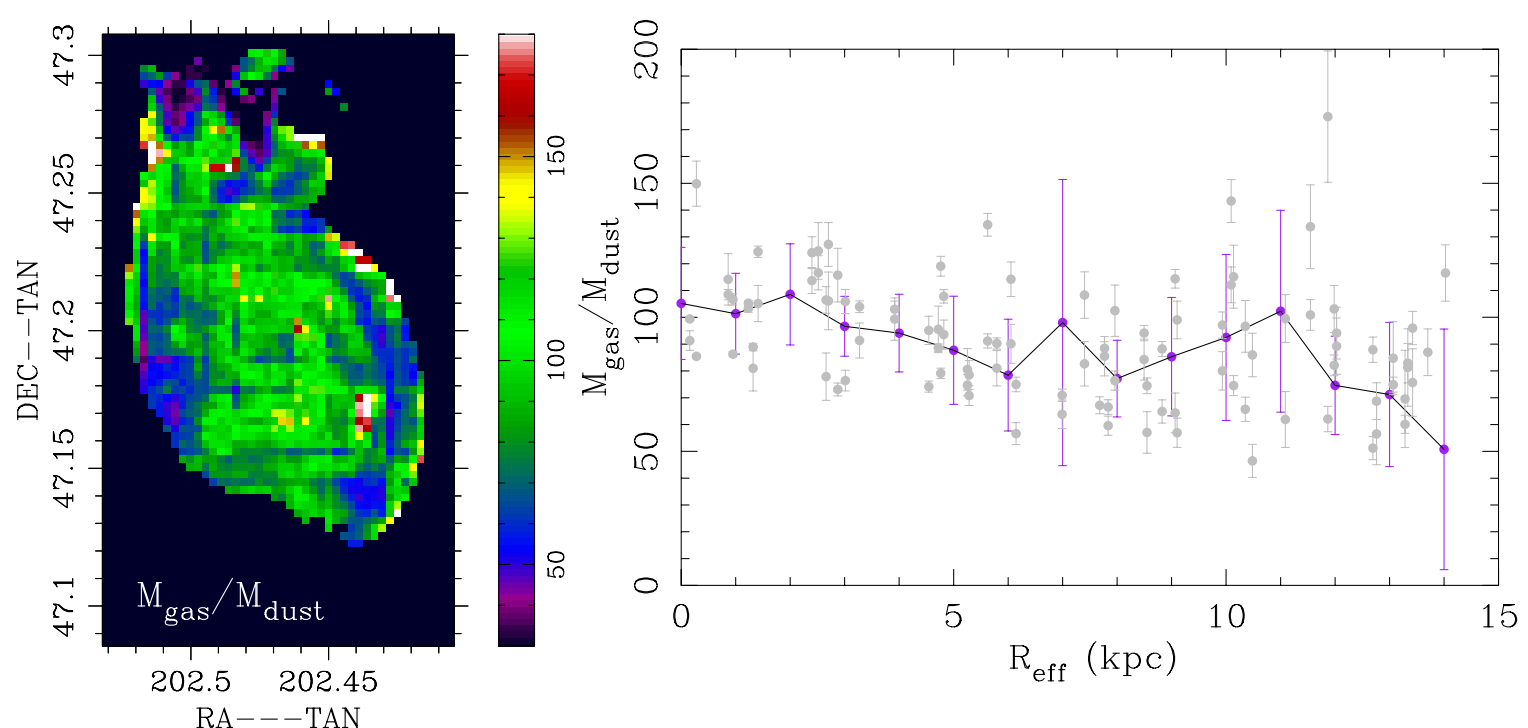

Figure 16. Gas-to-dust mass ratio map is shown on the left. The right shows the 1D profiles of the ratio as a function of radial distance from the center of NGC 5194 . The gray points show the pixel values and the connected line shows the binned radial profile.

(A color version of this figure is available in the online journal.)

Skibba et al. (2011) found to be $\log \left(M_{\text {dust }} / M_{\star}\right)=-2.70 \pm 0.53$, and NGC 5195 is in line with their findings for the earlytype galaxies in the survey with $\log \left(M_{\text {dust }} / M_{\star}\right)=-3.77 \pm$ 0.83 . Early-type galaxies in the volume-based HRS show the spheroid population has a lower dust-to-stellar mass ratio, with $\log \left(M_{\text {dust }} / M_{\star}\right)=-4.1 \pm 0.1$ (Smith et al. 2012), compared to NGC 5195 , although a couple of objects do have ratios as high as $\log \left(M_{\text {dust }} / M_{\star}\right)=-3.5$. For clarity, all the comparisons listed here have stellar and dust masses that were derived using a similar IMF (either a Chabrier 2003 or Kroupa 2001 IMF) and dust opacity and do not suffer from significant systematic offsets in the derived masses.

In comparison to larger volume-based infrared surveys, recent results from the H-ATLAS survey (Bourne et al. 2012) reveal that for blue galaxies there is a trend for the dust-to-stellar mass ratio to decrease with stellar mass. They adopt a higher opacity value than ours with $\kappa_{(\lambda=250 \mu \mathrm{m})}=0.89 \mathrm{~m}^{2} \mathrm{~kg}^{-1}$ compared with our choice of $\kappa_{(\lambda=250 \mu \mathrm{m})}=0.398 \mathrm{~m}^{2} \mathrm{~kg}^{-1}$. For comparison, their dust masses and dust-to-stellar mass ratios need to be shifted up by 0.35 dex. Stellar masses should be roughly consistent, as their chosen IMF (Chabrier 2003) leads to stellar masses only 0.03 dex lower than our chosen IMF from Kroupa (2001). Accounting for this, NGC 5194's dust-to-stellar mass ratio is consistent with the trend for blue galaxies from the H-ATLAS survey (with $\log \left(M_{\text {dust }} / M_{\star}\right) \sim-2.8$ ), while NGC 5195 is slightly lower, consistent with the green galaxies in their sample, which is the category it would belong to.

\subsubsection{Gas-to-dust Mass Ratio}

The map of the gas-to-dust ratio is shown in Figure 16, along with the 1D radial profile for NGC 5194 . The ratio is fairly constant across NGC 5194 and appears to slightly decrease radially from a value of $105 \pm 21$ in the inner $60^{\prime \prime}$ to a value of $71 \pm 27$ at a distance of $13 \mathrm{kpc}$ from the galaxy's center. Recalling the metallicity map of the system in Figure 7 and metallicity gradient results from Moustakas et al. (2010), the metallicity drops by a factor of $\sim 2$ from the central region to our aperture edge. Recent work by Magrini et al. (2011) and Foyle et al. (2012) show that a metallicity-dependent $X_{\mathrm{CO}}$ conversion factor is needed for regions dominated by molecular gas, which is the case for NGC 5194. Because of the radially decreasing metallicity, such a conversion would cause the gas-to-dust ratio to increase at larger radii, leading it to be even more constant across the galaxy as was seen for M83, also observed in the VNGS (Foyle et al. 2012).

In most cases, when a gradient is found in the gas-to-dust ratio, it often goes in the other direction, increasing with radial distance and is usually attributed to the galaxy containing a larger extended gas disk relative to its dust disk. This was seen in NGC 2403 (Bendo et al. 2010b) and in the sample of SINGS galaxies (Muñoz-Mateos et al. 2009). However, Muñoz-Mateos et al. (2009) actually find a fairly constant gas-to-dust ratio for NGC 5194 in agreement with our findings.

\subsection{Heating}

\subsubsection{Decoupling of Dust Mass and Heating}

There is a subtle decoupling between the distribution of the radiation intensities across the system and the dust mass surface density. Although both show spiral structure, when we superimpose them together as shown in the rightmost plot of Figure 17, it appears that there is a slight offset between the peaks of each quantity. This has been seen already in the nearby spiral M83 (Foyle et al. 2012), as well as in NGC 4501 and NGC 4567/8 in the Virgo Cluster (Smith et al. 2010). As in Foyle et al. (2012), we find the peaks in the ISRF (or dust temperature values) lie downstream of the spiral arm distribution of the dust, assuming a trailing arm rotation of NGC 5194 (counterclockwise).

There are two more obvious discrepancies between the $U_{\min }$ map and the dust mass map in the central regions of both galaxies. In the center of NGC 5194, there is an obvious increase in the dust mass density, relative to both the large-scale mass density as well as the mass density in the spiral arms. On the other hand, the ISRF is enhanced but only at the same level as the spiral arms. Even though the stellar, dust, and gas mass densities are all increasing, the local ISRF value stays moderately low. This is unlike the central region of NGC 5195, which has extremely high ISRF values (with peak dust temperatures of 

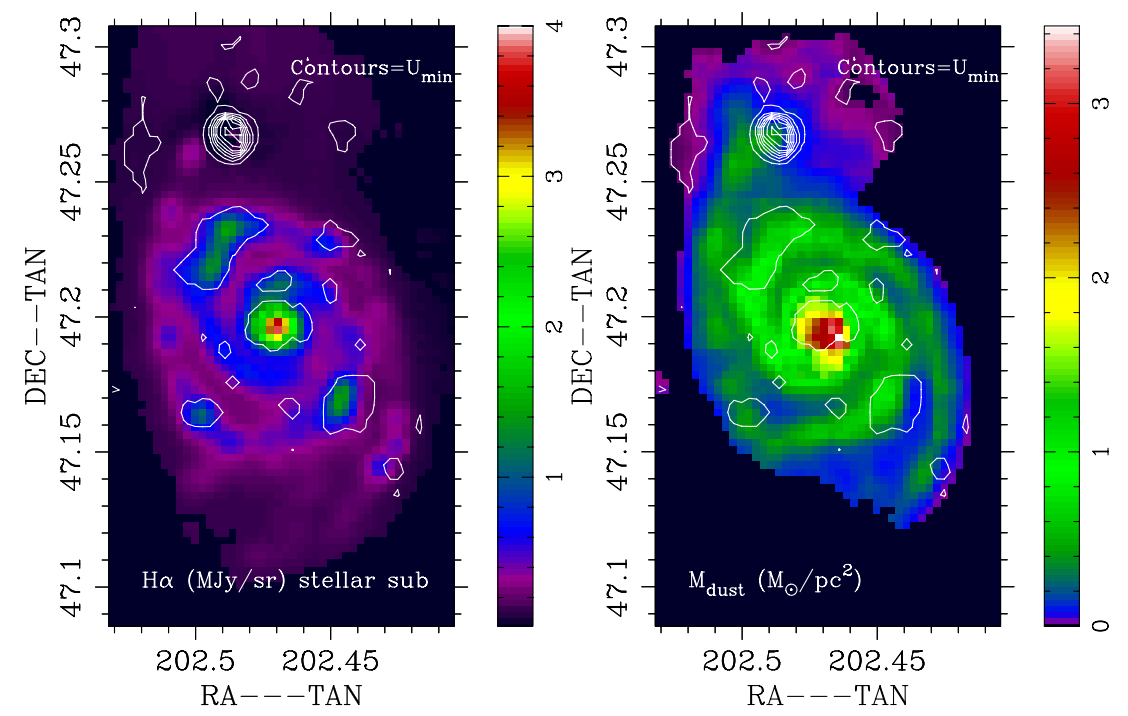

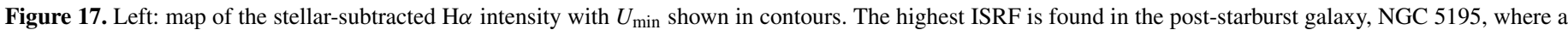

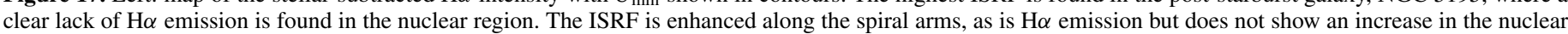

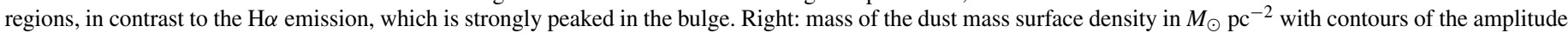

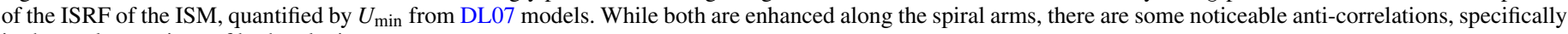
in the nuclear regions of both galaxies.

(A color version of this figure is available in the online journal.)

$30 \mathrm{~K}$ ), but no relative increase in the dust mass. Although there is less dust in the vicinity of NGC 5195, the dust that is there is quite warm. This is in agreement with measurements of the gas temperature by Kohno et al. (2002), who found that low $\mathrm{HCN}-\mathrm{CO}$ ratios suggest that the molecular gas in NGC 5195 is quite warm and unable to collapse and form stars.

\subsubsection{Ionized Gas and the ISRF}

In the left panel of Figure 17, we show the narrowband $\mathrm{H} \alpha$ image with stellar emission subtracted. This was done by calculating the stellar continuum transmitted through the $\mathrm{H} \alpha$ narrowband filter curve using the best-fit SED model without nebular emission included. The strength of the ISRF, as parameterized by $U_{\min }$, is shown in contours (and also in Figure 10). Qualitatively, the $\mathrm{H} \alpha$ emission and ISRF are spatially correlated in the disk of NGC 5194. They are both enhanced along the spiral arms and in the central bulge region, although $\mathrm{H} \alpha$ is more peaked than the ISRF in the nucleus perhaps due to AGN emission. In contrast, in NGC 5195, there is no detected star formation and hence no $\mathrm{H} \alpha$ emission. We note, however, that in optical spectroscopy from Moustakas et al. (2010), a modest amount of $\mathrm{H} \alpha$ emission is detected in the nuclear region at a $\sim 2 \sigma$ level that appears to be blueshifted relative to $\mathrm{H} \alpha$ absorption in stellar photospheres. Surprisingly, this is where the ISRF is the brightest in the entire map. In Figure 18 we show a 1D comparison between the ionized gas intensity and the strength of the ISRF determined from the dust emission model fitting. While the ionized gas intensity correlates with the ISRF in NGC 5194, it clearly follows another trend in NGC 5195 where high ISRF values correspond to no $\mathrm{H} \alpha$ emission.

\subsubsection{Dust Heating in NGC 5195}

One of the most interesting findings of this analysis is that the ISRF of the post-starburst spheroid NGC 5195 is up to 20-25 times the characteristic amplitude of the ISRF in the MW despite its lack of star formation. Often high dust temperatures are associated with starbursting and actively star-

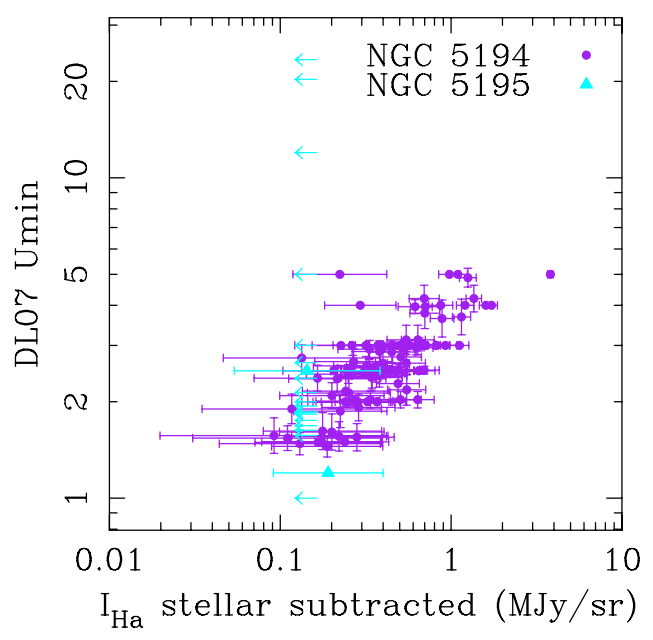

Figure 18. $\mathrm{H} \alpha$ emission is moderately correlated with the ISRF in NGC 5194 (purple points), while NGC 5195 (cyan triangles and upper limits) has minimal to zero $\mathrm{H} \alpha$ emission despite having high ISRF values.

(A color version of this figure is available in the online journal.)

forming galaxies. This is not the case for NGC 5195. The galaxy has a high $(29 \pm 3 \mathrm{~K})$ characteristic dust temperature in the nuclear region and an average temperature of $23 \pm 3 \mathrm{~K}$ in the galaxy's aperture. To provide some context, recent results from the H-ATLAS survey (Bourne et al. 2012) indicate that, for blue galaxies in their sample, dust temperatures increase with stellar mass up to a stellar mass of $\sim 6 \times 10^{10} M_{\odot}$ reaching a peak dust temperature of $\sim 25-30 \mathrm{~K}$ and then anti-correlate at higher stellar masses (see their Figure 14). NGC 5194, a blue galaxy according to their criteria, is consistent with this trend as is NGC 5195. However, NGC 5195 is not a blue galaxy, but is about 0.4 mag redder than NGC 5194 and would thus fall into their green galaxy color bin. In this color bin, a few galaxies exhibit high temperatures similar to NGC 5195, but their temperatures are poorly determined, likely because the dust model used to estimate the temperature was a poor fit to the observations. The 

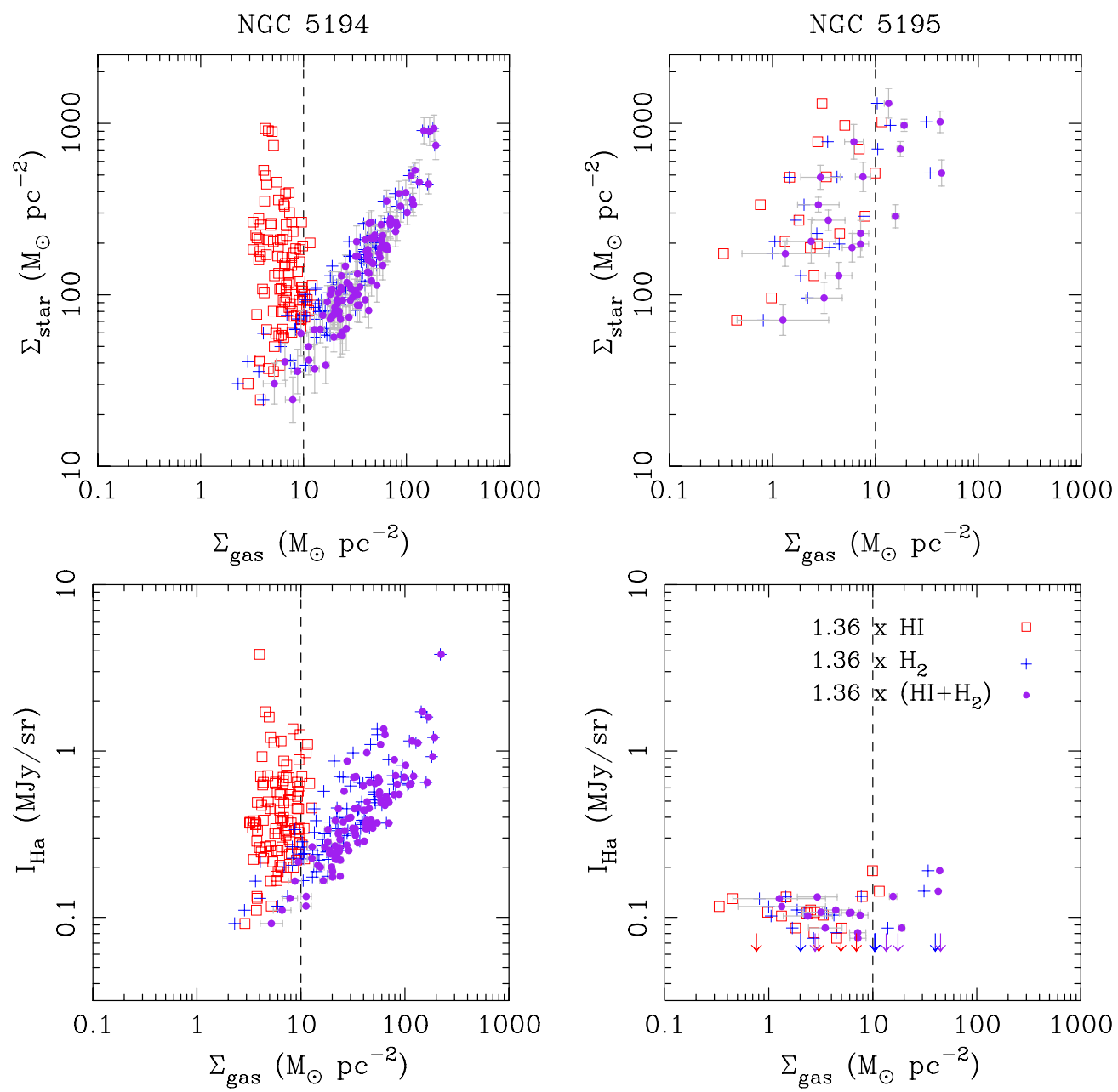

Figure 19. Stellar mass density vs. atomic (red squares), molecular (blue plus signs), and total (purple dots) gas mass surface density for NGC 5194 in the top left and for NGC 5195 in the top right. Stellar-subtracted $\mathrm{H} \alpha$ intensity as a function gas mass surface density is shown in the bottom panels. In each panel, we note the surface density threshold of $10 M_{\odot} \mathrm{pc}^{-2}$ at which atomic gas converts to molecular gas. Note that some regions in NGC 5195 contain molecular gas at densities lower than this threshold.

(A color version of this figure is available in the online journal.)

typical scenario suggests the redder the galaxy is, the cooler the dust temperature will be, but there are hints, particularly from the large scatter and uncertainty in constraining the temperature, that the transition from blue-to-red galaxy is more complex in the context of dust heating.

As a post-starburst galaxy, NGC 5195 is in a transition from starburst to quiescent. The lack of ionized gas (Thronson et al. 1991) indicates no recent star formation is ongoing but star formation peaked during its close encounter with NGC 5194 $\sim 400$ Myr ago. Other signposts that it is in a transition are evident by its low dust-to-stellar and gas-to-stellar mass ratios. Its gas fraction at $\sim 2 \%$ is very low and we show, in Figure 19, the gas that is in the galaxy appears to mostly be below the empirical threshold observed in the THINGS galaxy survey (Bigiel et al. 2008) at which atomic gas transitions to molecular gas, a requirement for star formation. In this figure, we show the stellar mass density as a function of gas mass density in the top panels, with NGC 5194 in the top left and NGC 5195 in the top right. The axes are kept on the same scale for each panel to show the different density parameter space in each galaxy. For a given stellar density, the gas density is considerably lower in NGC 5195 and there is a large scatter between stellar mass density and the total gas mass density (purple points). On the other hand, NGC 5194 shows a tighter relation between the total gas (purple) and stellar mass densities and there appears to be a threshold gas density of $\sim 10 M_{\odot} \mathrm{pc}^{-2}$ (marked by vertical dashed lines) at which the atomic gas (open red squares) is converted from neutral to molecular (blue plus signs). This threshold was noticed in the Kennicutt-Schmidt (K-S) relations drawn from the THINGS survey in Bigiel et al. (2008). In their plots, they used star formation density on the $y$-axis and found the same transition from atomic to molecular gas at a fixed gas density of $9 M_{\odot} \mathrm{pc}^{-2}$. In the bottom panel, we provide plots analogous to these K-S plots by plotting the intensity of $\mathrm{H} \alpha$ emission (a proxy for star formation rate density) in our narrowband images after subtracting stellar continuum based on our SED fitting. We do not correct for dust attenuation or convert the intensity to SFR density. The reader is referred to Bigiel et al. (2008), who already performed this analysis for NGC 5194. For NGC 5195, no star formation is found in the galaxy so no K-S relation is seen, in this galaxy.

So why is the dust temperature so high? We attempt to shed some light on the mechanism leading to the high temperature observed in this galaxy. Because this galaxy represents a nearby 

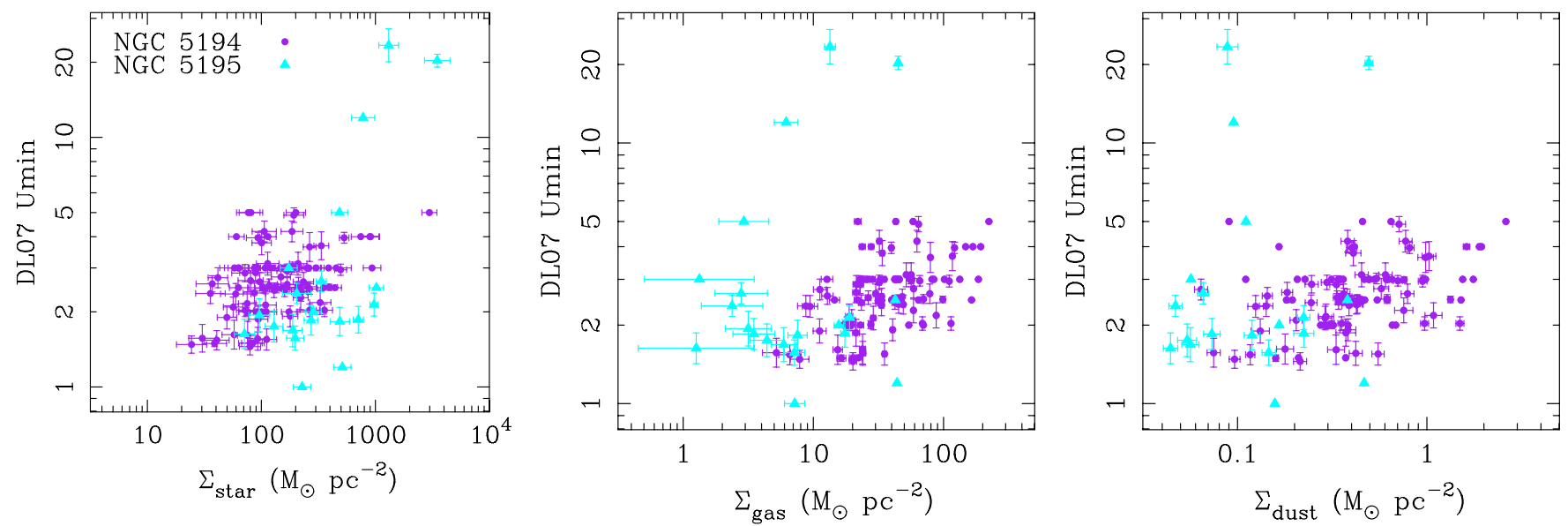

Figure 20. Amplitude of the ISRF as a function of stellar mass (left), total gas mass (middle), and dust (right) for NGC 5194 (purple dots) and NGC 5195 (cyan triangles). There is a modest trend for higher ISRF values to be found at higher stellar densities.

(A color version of this figure is available in the online journal.)

transitional galaxy, studying its resolved multi-wavelength properties can help isolate the source of heating. In the following we will explore some of the possible heating mechanisms: (1) heating from star formation, (2) heating from the evolved stellar population, and (3) heating by its AGN.

Heating by ongoing star formation. It is clear that the ionizing radiation of young massive stars is not heating the dust, as evident by its lack of ionized gas shown in Figure 18. The pixels in NGC 5195 are all upper limits in $\mathrm{H} \alpha$ so no ionized gas is detected except for a few regions near the edge of the galaxy, at which the emission from NGC 5195 begins to blend with the spiral and the local stellar density is lower.

Heating by the evolved population. Recent studies by Bendo et al. (2012) and Boselli et al. (2012) indicate that evolved stellar population heating plays a strong role in dust heating, although this is most important for lower temperature dust heating at longer infrared wavelength beyond $160 \mu \mathrm{m}$. In Figure 20, we plot the amplitude of the ISRF as a function of stellar, total gas, and dust mass densities. The ISRF does not correlate with either the stellar, dust, or gas mass density. In addition, the bulge in the spiral reaches similarly high stellar mass densities $\left(\sim 3 \times 10^{4} M_{\odot} \mathrm{pc}^{-2}\right)$ but has a much lower ISRF value compared with NGC 5195, so the enhanced stellar mass density cannot be the primary explanation for the enhanced ISRF observed in NGC 5195.

Heating by its active galactic nucleus. NGC 5195 has a compact radio source in the nucleus (van der Hulst et al. 1988) and has spectral indications of harboring an AGN (Moustakas et al. 2010). In a spectrum covering the nuclear region, a small amount of $\mathrm{H} \alpha$ emission is seen blueshifted relative to $\mathrm{H} \alpha$ seen in absorption, indicative of an outflow of ionized gas (Moustakas et al. 2010). In our observations, the ISRF is strongly peaked in the nuclear region and is suggestive that the AGN plays a role in heating the dust.

However, it is not completely clear that this is the dominant mechanism heating the dust and/or shutting down star formation. Mid-infrared observations with ISOCAM show a lack of AGN emission lines (Boulade et al. 1996) and rule out LINER activity as the dominant source of mid-infrared emission. Furthermore, NGC 5194 also harbors an AGN and based on equivalent widths and line intensities presented in Moustakas et al. (2010), the ionized emission associated with the AGN is
5-10 times brighter in NGC 5194. We caution that this comparison is complicated by unknown dust attenuation due to strong Balmer absorption in NGC 5195 (Ho et al. 1995). Unlike the nuclear region of NGC 5195, the ISRF is only slightly enhanced in the nuclear region in NGC 5194 (see the left panel in Figure 17), and at the same intensity as is seen in the spiral arms.

Unfortunately, none of these mechanisms stand out as the clear heating source. Although evolved population heating appears to be the most likely, with the other two almost ruled out. The stellar mass density is similar in both galaxies so we can only speculate that the relatively lower gas and dust fractions in NGC 5195 result in less efficient gas and dust cooling, leading to a relatively higher ISRF.

\subsubsection{PAH Destruction}

Looking back on Figure 10, the PAH fraction decreases slightly to $\sim 4 \%$ in the nuclear region of NGC 5194 due to a low-luminosity AGN (Moustakas et al. 2010). This is not too surprising. Many studies report (Genzel et al. 1998; Dale et al. 2006) the destruction of PAH molecules around AGNs and within hard radiation fields with PDRs (Boselli et al. 2004; Calzetti et al. 2005; Bendo et al. 2008). On the other hand, the decrease in PAH fraction in NGC 5195 is interesting. The PAH fraction traces the stellar mass surface density and decreases as a gradient across NGC 5195. One can see in the bottom-right SED of NGC 5195 in Figure 4 that PAH emission is easily detected and is many times the stellar emission at 5.8 and $8.0 \mu \mathrm{m}$. So the decrease in the mass fraction of PAHs relative to the total dust mass is not a detection issue. NGC 5195 also harbors an AGN (Moustakas et al. 2010) leading to a lower PAH fraction. But unlike NGC 5194, where the depression is concentrated in the nuclear region, the decrease in PAH fraction across NGC 5195 is a gradient. This may suggest NGC 5195 is accreting gas, dust, and PAH material from the nearby spiral arm, but as the PAHs approach the AGN, the fraction decreases.

\subsection{An Example of Morphological Quenching}

Unlike NGC 5194, the molecular gas in NGC 5195 is found at densities lower than $\sim 10 M_{\odot} \mathrm{pc}^{-2}$. If this threshold marks the density at which atomic gas becomes molecular, then it is likely that the molecular gas has come from NGC 5194 via 

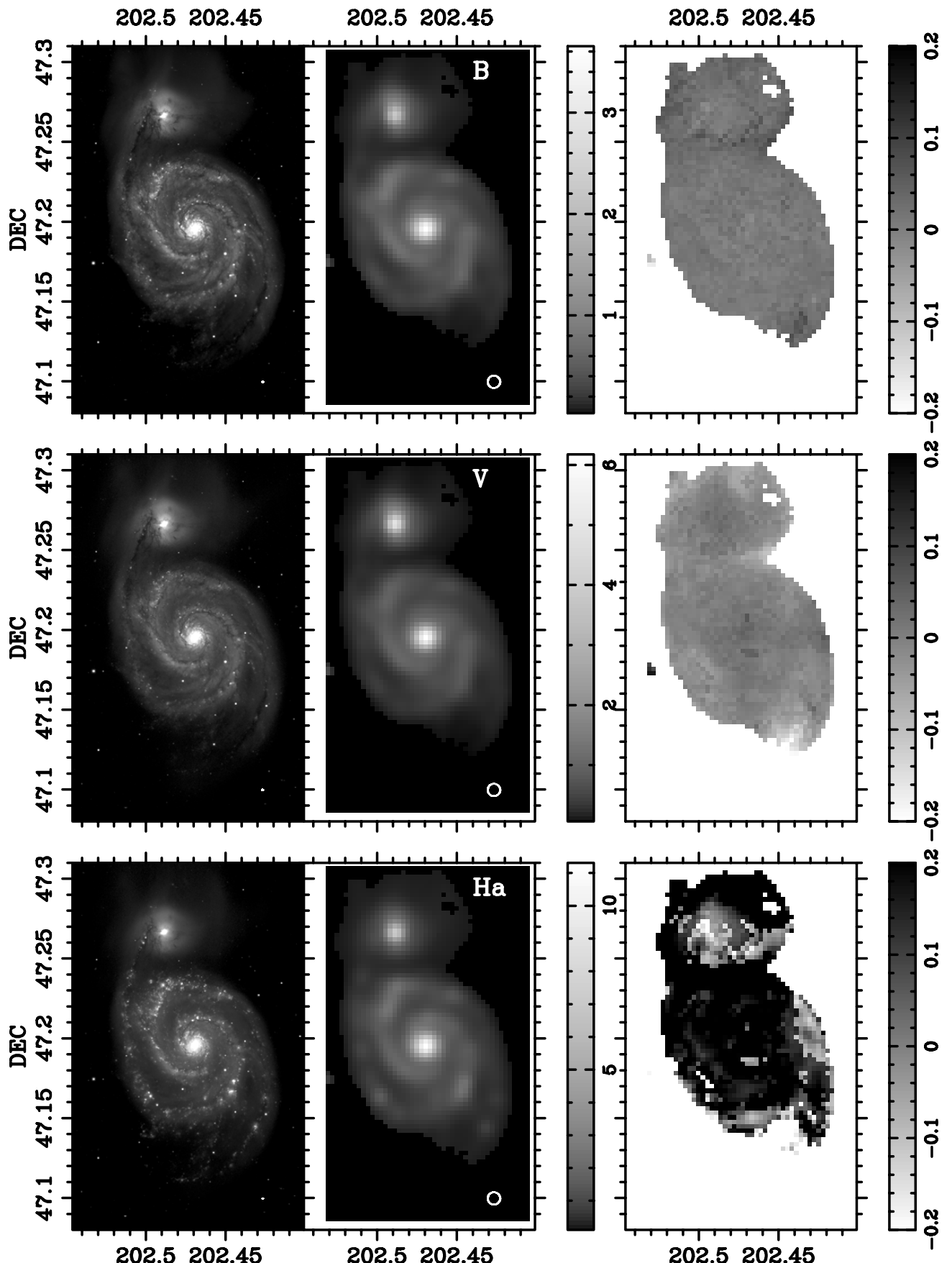

Figure 21. Optical images of NGC 5194/5195 from the SINGS survey (Kennicutt et al. 2003) from top to bottom: $B, V$, and H $\alpha$. The left panel is the original image, while the middle panel shows the image after matching it to a common resolution of $28^{\prime \prime}$ and plate scale of $10^{\prime \prime}$ pixel $^{-1}$. Both images are shown in MJy sr ${ }^{-1}$ and the beam area for each image is shown in the bottom right. For the left and middle panels, the intensity range (in $\mathrm{MJy} \mathrm{sr}^{-1}$ ) is indicated by the gradient on the right of the middle panel. The right column shows the residual image, defined in Equation (2), between the observed image after it is PSF matched (middle) and a synthetic galaxy image (not shown) generated by the best-fitting SED in our analysis. In the bottom row, the observed and processed H $\alpha$ narrowband image is shown (and includes stellar continuum emission in this band as there was no need to stellar continuum subtract in our analysis). The image was not included in our SED-fitting process, but we do show that overall the models-which include nebular emission-underestimate the amount of nebular emission by more than $20 \%$, except in NGC 5195, where the models accurately predict the amount of emission (mostly stellar continuum emission in this case) in the $\mathrm{H} \alpha$ bandpass.

accretion rather than being formed in situ. In addition to this, the molecular gas that does exist is unable to form stars because of the high stellar density (and thus its gravitational potential). The critical gas density required for the gas to cool and collapse is not satisfied. Modeled as a simple single-fluid Toomre stability model (Toomre 1964), Kennicutt (1989) shows that at higher stellar densities, the molecular gas must reach a higher critical density in order to cool and collapse. This is because localized shear motions from the gravitational potential are greater than the self-gravitational potential of the molecular cloud, thus making the gas stable. In regions where the average surface density of gas is less than five times the critical density, star formation is completely suppressed (Kennicutt 1989) because the molecular gas does not become dense enough to collapse into cold, dense molecular clouds required for star formation.

This scenario is supported by observations of the molecular gas in NGC 5195 by Kohno et al. (2002). They measured both $\mathrm{CO}(1-0)$ and $\mathrm{HCN}(1-0)$ in the galaxy and found an $\mathrm{HCN}$-to-CO 

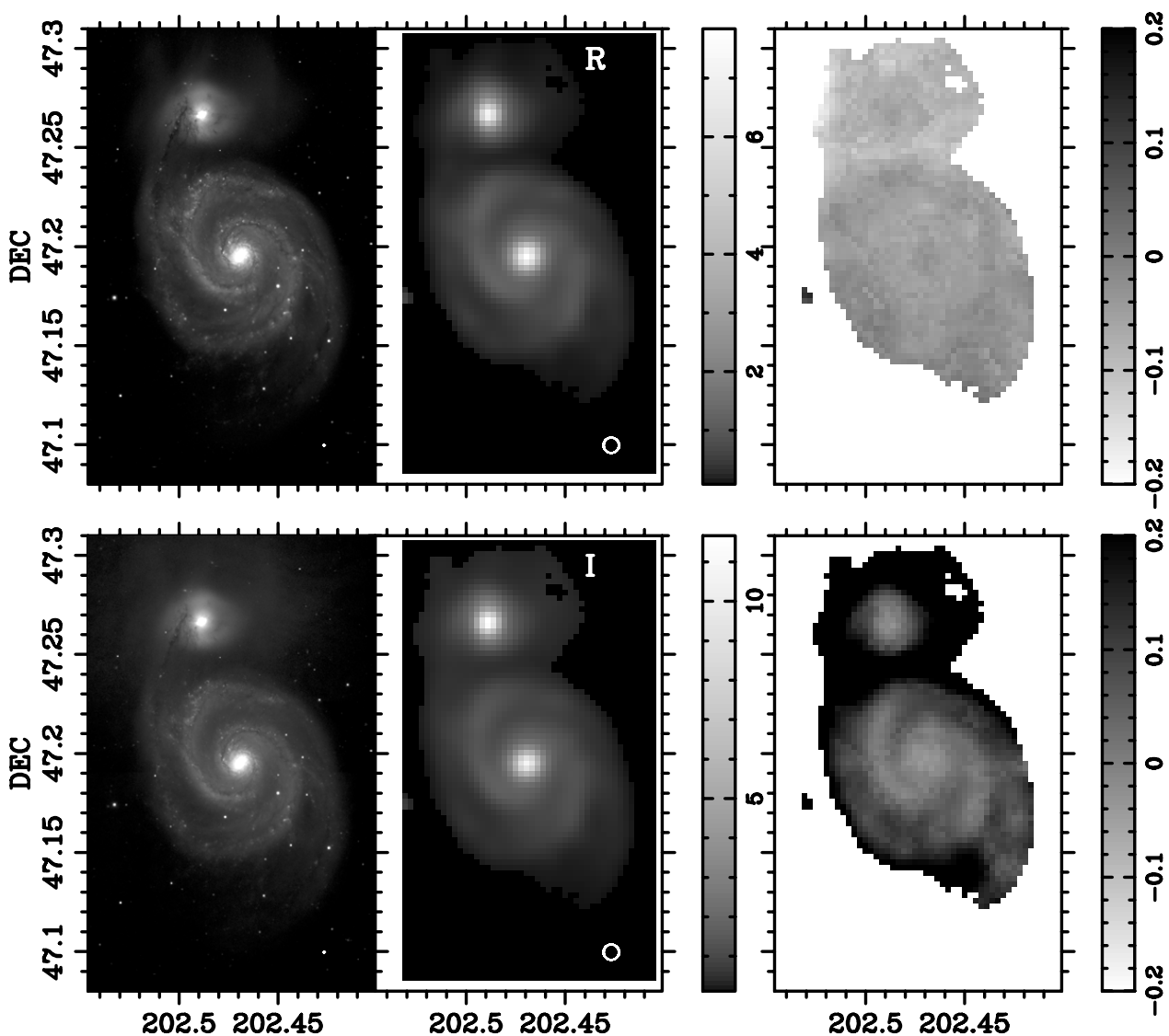

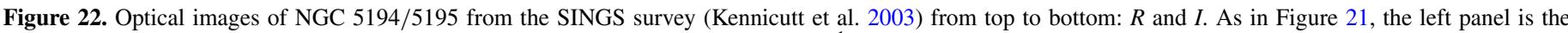

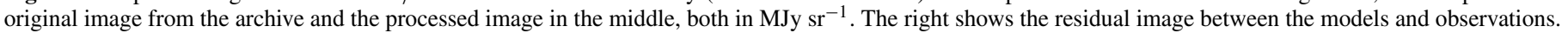

intensity ratio 5-15 times smaller than that seen in starburst regions (Helfer \& Blitz 1993; Kohno et al. 2002) and 5 times smaller than that usually found in spiral disks (Helfer \& Blitz 1995; Kohno et al. 1999), indicative of a lack of dense molecular cores.

Half a billion years ago, when the two galaxies were closer together, tidal forces would have disrupted such high shear velocities and likely involved higher rates of gas and dust accretion so the density required for clumps to form would have been lower and the gas supply available would have been more dense, allowing star formation to occur. But as the galaxies drew apart, the tidal forces were reduced and the dense molecular gas was exhausted as it was converted into stars during the galaxy's starburst episode.

This process, recently termed "morphological quenching" by Martig et al. (2009), occurs naturally within hydrodynamical simulations of galaxy evolution and can be extremely efficient under the right circumstances. Martig et al. (2009) showed that gaseous molecular disks, even those up to several $10^{9} M_{\odot}$, are unable to form stars if they are embedded within a sufficiently large gravitational potential such as a bulge-dominated galaxy. Star formation will cease even if gas accretion continues. The authors claim that this mode of star formation quenching is important for galaxies in dark matter halos less massive than $\sim 10^{12} M_{\odot}$ and thus an important mechanism for converting $L_{\star}$ and lower mass galaxies from the "blue cloud" to the "red sequence" and regulating galaxy growth, particularly because other quenching mechanisms such as AGN feedback or gas stripping are only effective in more massive halos. NGC 5195 provides a great nearby laboratory to further understand and validate this star formation quenching mechanism.

\section{CONCLUSIONS}

We have fit optical, near-, mid-, and far-infrared images of the Whirlpool galaxy system to a large library of stellar and dust SED models. By performing 100 Monte Carlo simulations of the photometry fitted to the models in each pixel, we have obtained maps of output parameters from stellar models generated by the SPS code PEGASE.2 (Fioc \& Rocca-Volmerange 1997) and dust model parameters generated by the dust emission model of Draine \& Li (2007). Our images were matched to a common plate scale and spatial resolution defined by a Gaussian PSF with an FWHM $=28^{\prime \prime}$ resolving the galaxy on physical scales of $\sim 1 \mathrm{kpc}$. Here we summarize the main conclusions of this work.

1. The SPS model fits reveal a burst of star formation occurred in both galaxies roughly 340-500 Myr ago, consistent with dynamical models of the interaction history of the galaxies (Salo \& Laurikainen 2000; Dobbs et al. 2010) as well as color-magnitude diagrams of individual stars (Tikhonov et al. 2009). There is little spatial dependence of the burst age and other SFH output parameters, suggesting a resolved SED approach is not necessary at this spatial resolution, corresponding to physical scales of $\sim 1 \mathrm{kpc}$. Metallicity and dust attenuation show some radial dependence but were not very well constrained by our method.

2. The dust-to-stellar mass ratio in NGC 5194 is $\log \left(M_{\text {dust }} / M_{\star}\right)=-2.5 \pm 0.2$, while NGC 5195 has a magnitude lower mass ratio at NGC 5195 at $\log \left(M_{\text {dust }} / M_{\star}\right)=$ $-3.5 \pm 0.3$. Both galaxies are consistent, according to galaxy type, with other galaxies observed with Herschel (Cortese et al. 2012; Smith et al. 2012; Bourne et al. 2012). 

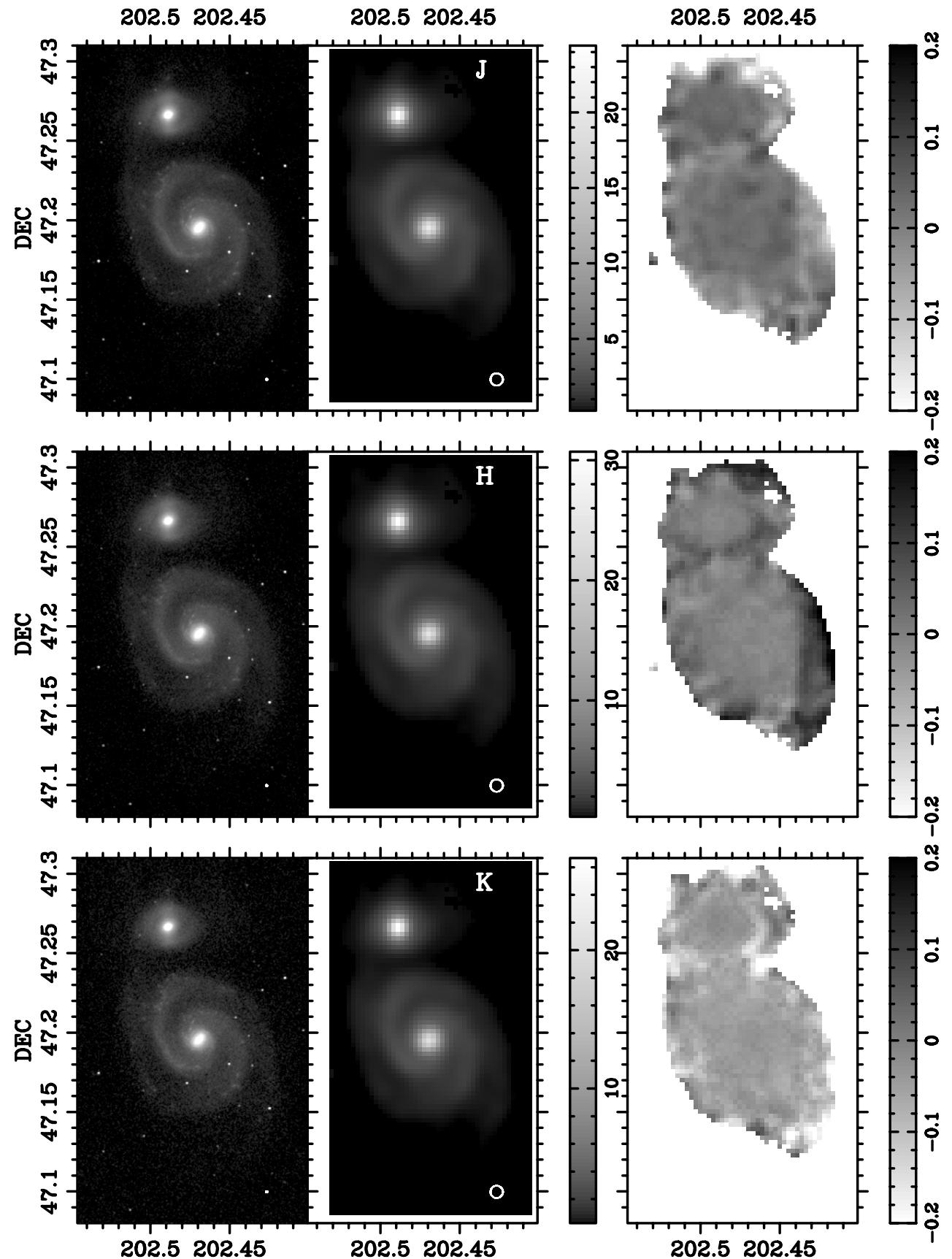

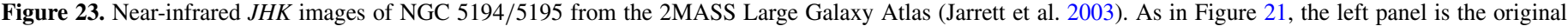
image from the archive and the processed image in the middle, both in $\mathrm{MJy} \mathrm{sr}^{-1}$. The right shows the residual image between the models and observations.

3. High radiation field values concentrated in the nuclear region of NGC 5195 provide evidence that the dust is located within the galaxy, not in its foreground. Despite a high ISRF (often an indication of star formation), there is no current star formation in the central region of NGC 5195 as indicated by a lack of $\mathrm{H} \alpha$ emission and lack of dense molecular clouds (Kohno et al. 2002).

4. Morphological quenching (Martig et al. 2009) is a plausible scenario for the suppression of star formation in NGC 5195. With its higher gravitational potential, the gas density threshold required for star formation is higher than the available low-density gas and is thus unable to form stars.

E.M.C. acknowledges the continued support for this research provided by the Natural Sciences and Engineering Research
Council (NSERC) of Canada through her PDF fellowship. This publication would not be possible without the legacy left by the great efforts of those involved and in the Spitzer Infrared Nearby Galaxies Survey (SINGS), the Two Micron All Sky Large Galaxy Atlas (2MASS LGA) and the BIMA SONG survey. This research was supported by grants from the Canadian Space Agency and the Natural Sciences and Engineering Research Council of Canada (PI: C. Wilson). PACS has been developed by a consortium of institutes led by MPE (Germany) and including UVIE (Austria); KU Leuven, CSL, IMEC (Belgium); CEA, LAM (France); MPIA (Germany); INAF-IFSI/OAA/OAP/ OAT, LENS, SISSA (Italy); IAC (Spain). This development has been supported by the funding agencies BMVIT (Austria), ESAPRODEX (Belgium), CEA/CNES (France), DLR (Germany), ASI/INAF (Italy), and CICYT/MCYT (Spain). SPIRE has been 

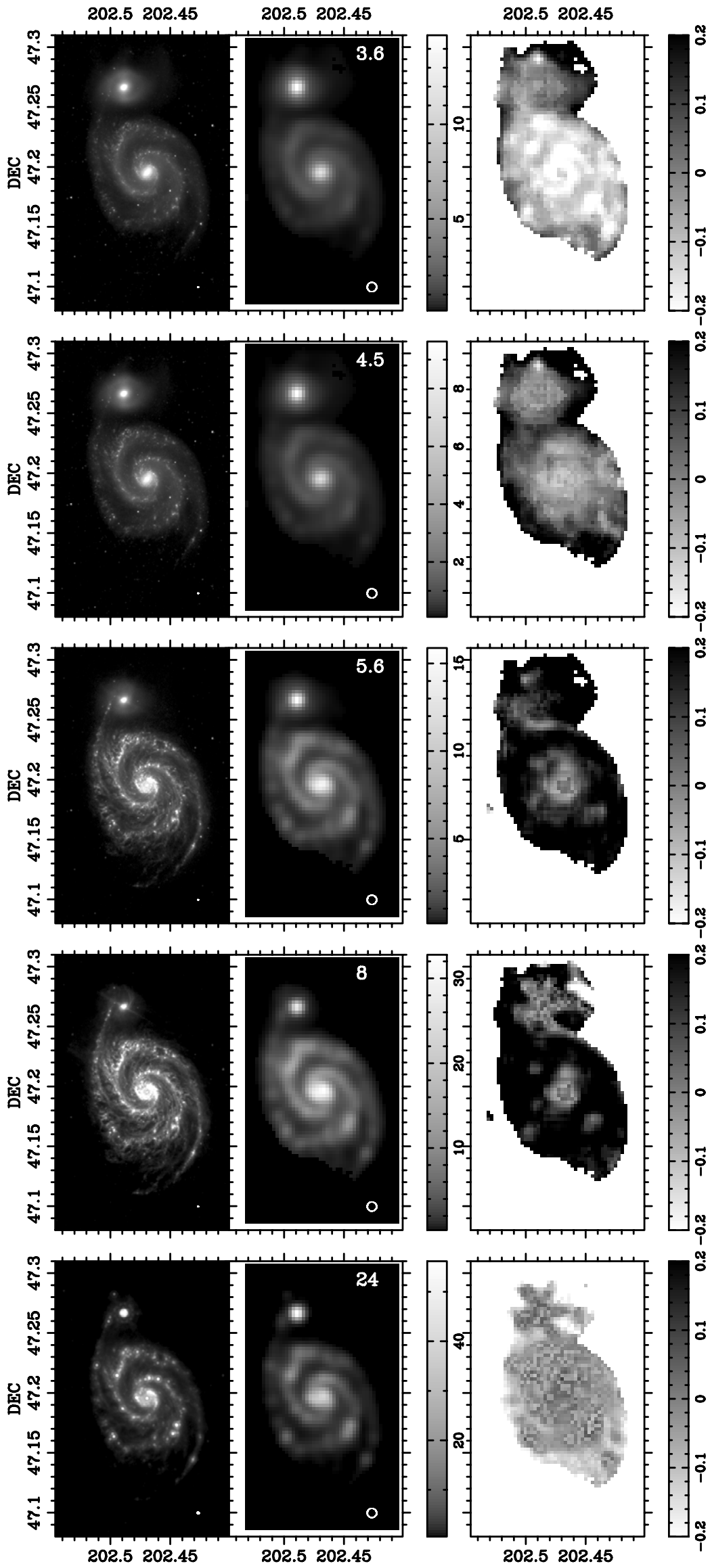

Figure 24. Mid-infrared images of NGC 5194/5195 from Spitzer as part of the SINGS survey (Kennicutt et al. 2003). As in Figure 21, the left panel is the original image from the archive and the processed image in the middle, both in $\mathrm{MJy} \mathrm{sr}^{-1}$. The right shows the residual image between the models and observations. The top two images were not used in either fit. 
developed by a consortium of institutes led by Cardiff University (UK) and including University of Lethbridge (Canada); NAOC (China); CEA, LAM (France); IFSI, University of Padua (Italy); IAC (Spain); Stockholm Observatory (Sweden); STFC and UKSA (UK); and Caltech, JPL, NHSC, University of Colorado (USA). This development has been supported by national funding agencies: CSA (Canada); NAOC (China); CEA, CNES, CNRS (France); ASI (Italy); MCINN (Spain); SNSB (Sweden); STFC (UK); and NASA (USA). HIPE is a joint development by the Herschel Science Ground Segment Consortium, consisting of ESA, the NASA Herschel Science Center, and the HIFI, PACS, and SPIRE consortia. This research has made use of the NASA/IPAC Extragalactic Database (NED) which is operated by the Jet Propulsion Laboratory, California Institute of Technology, under contract with the National Aeronautics and Space Administration.

\section{APPENDIX}

For reference, we show the image processing of the archival data used for our analysis. As described in Section 2, all images were matched to a common spatial resolution $\left(\mathrm{FWHM}=28^{\prime \prime}\right.$ ) and plate scale $\left(10^{\prime \prime}\right.$ pixel $\left.^{-1}\right)$. Figures 21 and 22 show the optical images taken from the ancillary catalog of the SINGS survey (Kennicutt et al. 2003). The left image is the original image of the galaxy at native resolution prior to any post-processing. The middle panel shows the galaxy after foreground stellar emission is replaced by emission in surrounding pixels. Following this, we are able to match the spatial resolution to the far-infrared images using convolution kernels as described in Section 2. The far right image shows a normalized residual map quantifying the difference between the observed and modeled galaxy band flux maps, as quantified in Equation (2).

Most of the optical images are well fit by the modes with the residual images revealing that the models fit the data to within $10 \%$, and thus within their uncertainties. The only image that is not well fit is the $\mathrm{H} \alpha$ image. This image was not used in the fitting routine but since the SEDs include nebular emission, ideally, it should be predicted by the SED models. The predicted nebular emission is reasonable in NGC 5195, which has no star formation, but in NGC 5194, the amount of $\mathrm{H} \alpha$ emission is underestimated. This is primarily because our chosen SFH is too simplistic and only modeled the very old population and the burst population from $\sim 400 \mathrm{Myr}$ ago. A more complex $\mathrm{SFH}$, such as a declining or constant star-forming burst, is needed to properly describe the recent star formation occurring in NGC 5194.

The near-infrared images from the 2MASS Large Galaxy Atlas (Jarrett et al. 2003) are shown in Figure 23. The models lead to small residual images, indicating a good fit to the NIR images. On the other hand, mid-infrared images are not as well fit, as shown in Figure 24. This is primarily because the mid-infrared images at 3.6 and $4.5 \mu \mathrm{m}$ are not used to fit the SED wavelengths and the modeled dust/PAH emission, extrapolated from longer wavelengths. Both models combined tend to overestimate the emission at 3.6 and $4.5 \mu \mathrm{m}$. More analysis is needed to determine whether the stellar or PAH emission is being overestimated or both. The 5.6 and $8.0 \mu \mathrm{m}$ images are used to fit the DL07 dust emission SED model. In the nuclear regions of both galaxies, the models are a good representation of the emission, but outside of these regions, the models underestimate the emission. Recalling from the PAH mass fraction map on the right in Figure 10 that in the regions outside of the nuclear region or edges of the galaxies, the PAH mass fraction reaches the highest value permitted by the DL07 models. This is likely the reason why the 5.6 and $8.0 \mu \mathrm{m}$ are underestimated by the models. A higher PAH fraction is needed to properly describe the observations. At longer wavelengths, just like our Herschel observations shown in Figure 2, the $24 \mu \mathrm{m}$ image is accurately described by the DL07 models.

\section{REFERENCES}

Aniano, G., Draine, B. T., Gordon, K. D., \& Sandstrom, K. 2011, PASP, 123, 1218

Asplund, M., Grevesse, N., Sauval, A. J., \& Scott, P. 2009, ARA\&A, 47, 481

Bendo, G. J., Boselli, A., Dariush, A., et al. 2012, MNRAS, 419, 1833

Bendo, G. J., Draine, B. T., Engelbracht, C. W., et al. 2008, MNRAS, 389, 629

Bendo, G. J., Wilson, C. D., Pohlen, M., et al. 2010a, A\&A, 518, L65

Bendo, G. J., Wilson, C. D., Warren, B. E., et al. 2010b, MNRAS, 402, 1409

Bigiel, F., Leroy, A., Walter, F., et al. 2008, AJ, 136, 2846

Boquien, M., Calzetti, D., Combes, F., et al. 2011, AJ, 142, 111

Boselli, A., Ciesla, L., Cortese, L., et al. 2012, A\&A, 540, A54

Boselli, A., Eales, S., Cortese, L., et al. 2010, PASP, 122, 261

Boselli, A., Lequeux, J., \& Gavazzi, G. 2004, A\&A, 428, 409

Boulade, O., Sauvage, M., Altieri, B., et al. 1996, A\&A, 315, L85

Bourne, N., Maddox, S. J., Dunne, L., et al. 2012, MNRAS, 421, 3027

Bresolin, F., Garnett, D. R., \& Kennicutt, R. C., Jr. 2004, ApJ, 615, 228

Bruzual, G., \& Charlot, S. 2003, MNRAS, 344, 1000

Calzetti, D., Kennicutt, R. C., Jr., Bianchi, L., et al. 2005, ApJ, 633, 871

Calzetti, D., Kinney, A. L., \& Storchi-Bergmann, T. 1994, ApJ, 429, 582

Chabrier, G. 2003, PASP, 115, 763

Charlot, S., \& Fall, S. M. 2000, ApJ, 539, 718

Compiègne, M., Verstraete, L., Jones, A., et al. 2011, A\&A, 525, A103

Conroy, C., Gunn, J. E., \& White, M. 2009, ApJ, 699, 486

Conroy, C., White, M., \& Gunn, J. E. 2010, ApJ, 708, 58

Cortese, L., Ciesla, L., Boselli, A., et al. 2012, A\&A, 540, A52

da Cunha, E., Charlot, S., \& Elbaz, D. 2008, MNRAS, 388, 1595

Dale, D. A., \& Helou, G. 2002, ApJ, 576, 159

Dale, D. A., Smith, J. D. T., Armus, L., et al. 2006, ApJ, 646, 161

Désert, F.-X., Boulanger, F., \& Puget, J. L. 1990, A\&A, 237, 215

de Vaucouleurs, G., de Vaucouleurs, A., Corwin, H. G., et al. 1995, VizieR

Online Data Catalog, 7155, 0

Devriendt, J. E. G., Guiderdoni, B., \& Sadat, R. 1999, A\&A, 350, 381

Dobbs, C. L., Theis, C., Pringle, J. E., \& Bate, M. R. 2010, MNRAS, 403, 625

Draine, B. T. 2003, ARA\&A, 41, 241

Draine, B. T., Dale, D. A., Bendo, G., et al. 2007, ApJ, 663, 866

Draine, B. T., \& Li, A. 2007, ApJ, 657, 810

Feldmeier, J. J., Ciardullo, R., \& Jacoby, G. H. 1997, ApJ, 479, 231

Fioc, M., \& Rocca-Volmerange, B. 1997, A\&A, 326, 950

Foyle, K., Wilson, C. D., Mentuch, E., et al. 2012, MNRAS, 421, 2917

Galliano, F., Hony, S., Bernard, J.-P., et al. 2011, A\&A, 536, A88

Genzel, R., Lutz, D., Sturm, E., et al. 1998, ApJ, 498, 579

Glazebrook, K., Abraham, R. G., McCarthy, P. J., et al. 2004, Nature, 430, 181

Griffin, M. J., Abergel, A., Abreu, A., et al. 2010, A\&A, 518, L3

Helfer, T. T., \& Blitz, L. 1993, ApJ, 419, 86

Helfer, T. T., \& Blitz, L. 1995, ApJ, 450, 90

Ho, L. C., Filippenko, A. V., \& Sargent, W. L. 1995, ApJS, 98, 477

Indebetouw, R., Mathis, J. S., Babler, B. L., et al. 2005, ApJ, 619, 931

Jarrett, T. H., Chester, T., Cutri, R., Schneider, S. E., \& Huchra, J. P. 2003, AJ, 125,525

Kennicutt, R. C., Jr. 1989, ApJ, 344, 685

Kennicutt, R. C., Jr., Armus, L., Bendo, G., et al. 2003, PASP, 115, 928

Kennicutt, R. C., Calzetti, D., Aniano, G., et al. 2011, PASP, 123, 1347

Kobulnicky, H. A., \& Kewley, L. J. 2004, ApJ, 617, 240

Koda, J., Sawada, T., Wright, M. C. H., et al. 2011, ApJS, 193, 19

Kohno, K., Kawabe, R., \& Vila-Vilaró, B. 1999, ApJ, 511, 157

Kohno, K., Tosaki, T., Matsushita, S., et al. 2002, PASJ, 54, 541

Kroupa, P. 2001, MNRAS, 322, 231

Lee, J. H., Hwang, N., \& Lee, M. G. 2011, ApJ, 735, 75

Magrini, L., Bianchi, S., Corbelli, E., et al. 2011, A\&A, 535, A13

Martig, M., Bournaud, F., Teyssier, R., \& Dekel, A. 2009, ApJ, 707, 250

Mentuch, E., Abraham, R. G., Glazebrook, K., et al. 2009, ApJ, 706, 1020

Mentuch, E., Abraham, R. G., \& Zibetti, S. 2010, ApJ, 725, 1971

Moustakas, J., Kennicutt, R. C., Jr., Tremonti, C. A., et al. 2010, ApJS, 190, 233

Muñoz-Mateos, J. C., Gil de Paz, A., Zamorano, J., et al. 2009, ApJ, 703, 1569

Muzzin, A., Marchesini, D., van Dokkum, P. G., et al. 2009, ApJ, 701, 1839

Noll, S., Burgarella, D., Giovannoli, E., et al. 2009, A\&A, 507, 1793 
Pei, Y. C. 1992, ApJ, 395, 130

Pilyugin, L. S., \& Thuan, T. X. 2005, ApJ, 631, 231

Poglitsch, A., Waelkens, C., Geis, N., et al. 2010, A\&A, 518, L2

Popescu, C. C., Tuffs, R. J., Dopita, M. A., et al. 2011, A\&A, 527, A109

Salo, H., \& Laurikainen, E. 2000, MNRAS, 319, 377

Schlegel, D. J., Finkbeiner, D. P., \& Davis, M. 1998, ApJ, 500, 525

Skibba, R. A., Engelbracht, C. W., Dale, D., et al. 2011, ApJ, 738, 89

Smith, M. W. L., Gomez, H. L., Eales, S. A., et al. 2012, ApJ, 748, 123

Smith, M. W. L., Vlahakis, C., Baes, M., et al. 2010, A\&A, 518, L51

Strong, A. W., Bloemen, J. B. G. M., Dame, T. M., et al. 1988, A\&A, 207, 1
Swinyard, B. M., Ade, P., Baluteau, J.-P., et al. 2010, A\&A, 518, L4

Terashima, Y., \& Wilson, A. S. 2001, ApJ, 560, 139

Thronson, H. A., Jr., Rubin, H., \& Ksir, A. 1991, MNRAS, 252, 550

Tikhonov, N. A., Galazutdinova, O. A., \& Tikhonov, E. N. 2009, Astron. Lett., 35,599

Toomre, A. 1964, ApJ, 139, 1217

van der Hulst, J. M., Kennicutt, R. C., Crane, P. C., \& Rots, A. H. 1988, A\&A, 195,38

Walter, F., Brinks, E., de Blok, W. J. G., et al. 2008, AJ, 136, 2563

Weingartner, J. C., \& Draine, B. T. 2001, ApJ, 548, 296

Wilson, C. D., Warren, B. E., Israel, F. P., et al. 2009, ApJ, 693, 1736 